\title{
The Physical Drivers of the Luminosity-weighted Dust Temperatures in High-redshift Galaxies
}

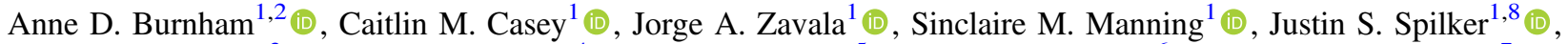 \\ Scott C. Chapman ${ }^{3}$ (i), Chian-Chou Chen ${ }^{4}$ (i), Asantha Cooray ${ }^{5}$ (i), David B. Sanders ${ }^{6}$ (1) , and Nick Z. Scoville ${ }^{7}$ (1) \\ ${ }^{1}$ Department of Astronomy, The University of Texas at Austin, 2515 Speedway Boulevard, Stop C1400, Austin, TX 78712, USA; anne.burnham@yale.edu \\ ${ }^{2}$ Department of Astronomy, Yale University, 52 Hillhouse Avenue, New Haven, CT 06511, USA \\ ${ }^{3}$ Department of Physics and Atmospheric Science, Dalhousie University, 6310 Coburg Road, Halifax, Nova Scotia, B3H 4R2, Canada \\ ${ }^{4}$ Academia Sinica Institute of Astronomy and Astrophysics (ASIAA), No. 1, Section 4, Roosevelt Road, Taipei 10617, Taiwan \\ ${ }^{5}$ Center for Cosmology, Department of Physics and Astronomy, University of California, Irvine, CA 92697, USA \\ ${ }^{6}$ Institute for Astronomy, University of Hawaii at Manoa, 2680 Woodlawn Drive, Honolulu, HI 96822, USA \\ ${ }^{7}$ California Institute of Technology, 1216 East California Boulevard, Pasadena, CA 91125, USA \\ Received 2020 May 12; revised 2021 January 25; accepted 2021 February 4; published 2021 March 30
}

\begin{abstract}
The underlying distribution of galaxies' dust spectral energy distributions (SEDs) (i.e., their spectra reradiated by dust from rest-frame $\sim 3 \mu \mathrm{m}$ to $3 \mathrm{~mm}$ ) remains relatively unconstrained owing to a dearth of far-IR/(sub)millimeter data for large samples of galaxies. It has been claimed in the literature that a galaxy's dust temperature-observed as the wavelength where the dust SED peaks $\left(\lambda_{\text {peak }}\right)$ - is traced most closely by its specific star formation rate (sSFR) or parameterized "distance" to the SFR- $M_{\star}$ relation (the galaxy "main sequence"). We present 0 ". 24 resolved $870 \mu \mathrm{m}$ ALMA dust continuum observations of seven $z=1.4-4.6$ dusty star-forming galaxies chosen to have a large range of well-constrained luminosity-weighted dust temperatures. We also draw on similar-resolution dust continuum maps from a sample of ALESS submillimeter galaxies from Hodge et al (2016). We constrain the physical scales over which the dust radiates and compare those measurements to characteristics of the integrated SED. We confirm significant correlations of $\lambda_{\text {peak }}$ with both $L_{\mathrm{IR}}$ (or SFR) and $\Sigma_{\mathrm{IR}}(\propto \mathrm{SFR}$ surface density). We investigate the correlation between $\log _{10}\left(\lambda_{\text {peak }}\right)$ and $\log _{10}\left(\Sigma_{\mathrm{IR}}\right)$ and find the relation to hold as would be expected from the StefanBoltzmann law, or the effective size of an equivalent blackbody. The correlations of $\lambda_{\text {peak }}$ with sSFR and distance from the SFR $-M_{\star}$ relation are less significant than those for $\Sigma_{\mathrm{IR}}$ or $L_{\mathrm{IR}}$; therefore, we conclude that the more fundamental tracer of galaxies' luminosity-weighted integrated dust temperatures are indeed their star formation surface densities in line with local universe results, which relate closely to the underlying geometry of dust in the interstellar medium.
\end{abstract}

Unified Astronomy Thesaurus concepts: Galaxy evolution (594); Dust continuum emission (412); Starburst galaxies (1570); High-redshift galaxies (734); Submillimeter astronomy (1647)

\section{Introduction}

Dusty star-forming galaxies (DSFGs) have incredibly high star formation rates (SFRs) and in the first few gigayears produce $\sim 50 \%$ of the stellar mass in the universe (Casey et al. 2012; Gruppioni et al. 2013). They are vitally important to galaxy evolution, but many of their fundamental dust characteristics are not well studied. Though their far-IR (FIR) through millimeter spectral energy distributions (SEDs) are relatively straightforward to interpret as a linear combination of modified blackbodies from dust, which reradiates nascent starlight, the lack of detailed photometry along that SED (rarely exceeding a few measurements across a $1000 \mu \mathrm{m}$ range) has limited our understanding of the physics governing that dust in the interstellar medium (ISM). This includes the physical scales over which the dust radiates and how clumpy it is, perhaps tracing back to its origins in/around compact star clusters. Physically tracing the relationship between integrated SEDs and underlying geometry is critically important.

A major challenge in characterizing galaxies' dusty SEDs has been the fundamental limitation of infrared through radio data sets, particularly for high- $z$ galaxies. For $10 \mathrm{yr}$ before 2010 most analysis was limited to SCUBA flux densities along the RayleighJeans (RJ) tail of blackbody emission, which made it impossible to constrain both the IR luminosity and dust temperature of a

\footnotetext{
${ }^{8}$ Hubble Fellow.
}

given high- $z$ galaxy. Even the vast improvement ushered in by Herschel in the past decade has fundamental limitations given the telescope's large beam size at $250-500 \mu \mathrm{m}$, where SEDs of $z=1-3$ galaxies peak. The uncertainty brought on by confusion noise, added with the relatively shallow depth of Herschel surveys, provides some moderate breakthroughs in measuring galaxies' dust temperatures across cosmic time (e.g., Lee et al. 2013; Symeonidis et al. 2013).

Dust temperature is observationally constrained through measurement of $\lambda_{\text {peak }}$, the wavelength at which the SED peaks in the rest frame, which is inversely proportional to the underlying physical dust temperature via Wien's law (Wien 1897). The precise mapping of $\lambda_{\text {peak }}$ to $T_{\mathrm{d}}$ depends on the underlying opacity of the dust in the ISM (see Figure 20 of Casey et al. 2014), and thus $T_{\mathrm{d}}$ is usually unconstrained without spatially resolved observations. Empirical data sets have shown us that DSFGs at all epochs have higher temperatures at higher IR luminosities (e.g., Perault 1987; Blain et al. 2004; Casey et al. 2014). This relationship is akin to a Stefan-Boltzmann law for the cold ISM on galaxy scales even without direct accounting for sources' emitting regions or underlying dust opacity. Indeed, previous studies in the local universe see a relationship between the SFR surface density, $\Sigma_{\mathrm{IR}}$, and integrated galaxy SED dust temperatures (Chanial et al. 2007; Lutz et al. 2016) as would be expected given a Stefan-Boltzmann-type relation. This physical reasoning has 
also been used to interpret the integrated SEDs of high-redshift dusty galaxies (e.g., Hodge et al. 2016; Simpson et al. 2017), though some discrepancies in interpretation remain.

Trends in galaxies' dust temperatures at high redshifts have been measured frequently in the literature with somewhat mixed, potentially contradictory results. Some suggest evolution toward colder dust temperatures for higher-redshift systems (Casey et al. 2012; Lee et al. 2013; Symeonidis et al. 2013; Kirkpatrick et al. 2017), while others suggest evolution toward warmer dust temperatures at higher redshifts (e.g., Magdis et al. 2012; Magnelli et al. 2014; Béthermin et al. 2015, 2017; Schreiber et al. 2018). All of these works attempt to quantify the underlying physical drivers of galaxies' bulk dust temperatures. We describe the basis of these claims and how they are not actually contradictory to one another in a broader context in Section 2 of this paper. We use this diverse range of claims, and the physics used to explain them, as motivation for this study.

In this paper, we have selected a sample of seven DSFGs with a wide range of measured dust temperatures and confirmed spectroscopic redshifts for high spatial resolution submillimeter follow-up to directly test for correlation between dust temperature and other galaxy characteristic quantities. We use new resolved dust continuum maps from ALMA band 7 observations at $870 \mu \mathrm{m}$ of these seven DSFGs to investigate the hypothesis that dust temperature maps directly to other observable characteristics such as galaxy size, morphology, star formation surface density, and specific SFR (sSFR). We also include 11 ALESS sources in our analysis from Hodge et al. (2016), whose observations and sample are similar, though their sources were $1.3 \times$ brighter and observations were slightly shallower. Section 2 provides background from the literature, giving more in-depth motivation for this investigation. Section 3 describes sample selection and observations. Section 4 gives our calculations of key physical properties of the relevant DSFGs. In Section 5 we discuss our findings, and we provide our conclusions in Section 6. We assume a concordance cosmology throughout this paper, adopting $H_{0}=70 \mathrm{~km} \mathrm{~s}^{-1} \mathrm{Mpc}, \Omega_{M}=0.3$, and $\Omega_{\Lambda}=0.7$. Where SFRs are used, we assume a Kroupa initial mass function (IMF; Kroupa $\&$ Weidner 2003) and scaling relations drawn from Kennicutt \& Evans (2012).

\section{Context of Galaxies' Dust Temperatures}

We draw motivation for this detailed study of a subsample of galaxies with high-quality resolved millimeter dust maps from the many discussions presented in the literature of trends in galaxies' overall dust temperatures with redshift. This section provides context of these discussions.

Dust is heated by a radiation field with an intensity represented by $U$. The dust is heated in two environments: the ambient radiation field heating the diffuse ISM, and discrete photon heating within star-forming regions. The diffuse ISM portion is heated by a radiation field with constant $U_{\text {min }}$, and the other portion is heated by radiation (primary and secondary photons) from young stars with intensities ranging from $U_{\min }$ and $U_{\max }$ (Magdis et al. 2012). The equation governing the dust-weighted mean starlight intensity scale factor, $\langle U\rangle$, is defined in Magdis et al. (2012). From this equation, $\langle U\rangle$ is proportional to $L_{\mathrm{IR}} / M_{\text {dust }}$. A corollary is that the luminosity-weighted dust temperature correlates directly with $\langle U\rangle$. Dust temperature increases with the strength of the radiation field. Similarly, a more compact distribution of dust around the source of incident radiation increases the dust temperature.

Magdis et al. (2012) used samples of Herschel-detected galaxies to argue that the galaxy IR SED depends solely on $\langle U\rangle$ and is independent of sSFR and $M_{\star}$. They also argue that $\langle U\rangle$ evolves with time such that main-sequence galaxies at earlier epochs had more intense radiation fields, or higher $\langle U\rangle$. They find that $T_{\mathrm{d}}$ evolves with time such that main-sequence galaxies at higher $z$ (out to $z \sim 2$ ) have warmer temperatures than those at $z=0$. Note that this is not necessarily contradictory to the finding that $z \sim 2$ galaxies have colder SEDs than $z \sim 0$ galaxies of similar $L_{\mathrm{IR}}$; this is due to the dramatic evolution in the main sequence between these epochs.

Magnelli et al. (2014) used Herschel observations to propose stronger correlations of $T_{\mathrm{d}}$ with $\mathrm{SSFR}$ and parameterized distance to the main sequence $\left(D_{\mathrm{MS}}\right)$ rather than with $L_{\mathrm{IR}}$ explicitly. The correlation is such that dust temperature is fixed for a fixed redshift and sSFR, implying that galaxies with a particular sSFR contain star-forming regions with similar $\langle U\rangle$. An increase in these star-forming regions results in the increase of SFR with stellar mass $\left(M_{\star}\right)$. Since starbursts have higher SFRs than galaxies with equivalent masses on the main sequence, more intense radiation fields coupled with higher densities could cause the elevated dust temperatures (Magnelli et al. 2014).

A number of additional works also find an increasing dust temperature for main-sequence galaxies, including Béthermin et al. (2017) and Schreiber et al. (2018), among others. Magdis et al. (2012) and Béthermin et al. (2015) assert that the evolution of $\langle U\rangle$ points to an evolution of $T_{\mathrm{d}}$ for all galaxies on the main sequence. Magdis et al. (2012) and Schreiber et al. (2018) posit that the evolution of main-sequence galaxy SEDs with redshift proves an evolution in $T_{\mathrm{d}}$.

In contrast to the works that find an evolution toward hotter dust temperatures for main-sequence galaxies at higher redshifts, several papers report that high-redshift galaxies evolve toward colder temperatures at higher redshifts, in particular at $z \sim 2$ relative to galaxies at $z \sim 0$. Such colder temperatures have been inferred based on galaxies of fixed SFR or $L_{\mathrm{IR}}$ and credited possibly to more extended dust geometries in high-redshift galaxies (e.g., Casey et al. 2012; Symeonidis et al. 2013). However, Casey et al. (2018a) argue that this perceived evolution toward colder temperatures could, in part, be a bias in underlying data sets that exist for $z \sim 0$ dust SEDs versus those at $z \sim 2$, and that most of the evolution, if it does exist, is between $0<z<0.4$. Further work is needed to understand whether this very low redshift evolution is physically real or purely driven by limits in the existing data sets (P. M. Drew et al. 2021 , in preparation).

Beyond $z \sim 0.5$ and out to $z \sim 5$, Casey et al. (2018b) find no evidence for redshift evolution of galaxies' SEDs in the $L_{\mathrm{IR}}-\lambda_{\text {peak }}$ plane. Similarly, Dudzevičiūtè et al. (2020) find no evidence for evolution in dust temperature for galaxies as a function of fixed $L_{\mathrm{IR}}$. How can these seemingly disparate conclusions-that SEDs of main-sequence galaxies evolve, yet there is no observed evolution in $L_{\mathrm{IR}}-\lambda_{\text {peak }}$ with $z$-be reconciled? These results are not, in fact, contradictory. In the main sequence, SFR (or $L_{\mathrm{IR}}$ ) evolves with $z$, but $\lambda_{\text {peak }}$ does not evolve at fixed SFR. A direct correlation between $L_{\mathrm{IR}}$ and $\lambda_{\text {peak }}$, or dust temperature, is well established (e.g., Chapman et al. 2003a; Sanders et al. 2003; Casey et al. 2018b) and has been shown to be driven by increased dust heating in more luminous systems (Symeonidis et al. 2013) 
and to be independent of luminosity-limit selection effects (Sajina et al. 2007; Lee et al. 2013). As objects in the main sequence evolve toward higher luminosities (and correspondingly higher $\langle U\rangle$ ) with $z, \lambda_{\text {peak }}$ appears to evolve with redshift at fixed $M_{\star}$ owing to its correlation with $L_{\mathrm{IR}}$. Thus, the perceived $T_{\mathrm{d}}-z$ evolution holds only for fixed sSFR, $D_{\mathrm{MS}}$, or $M_{\star}$, and not for fixed SFR.

In this paper, we explore the dependence of $\lambda_{\text {peak }}$ on SFR, sSFR, star formation surface density, and parameterized distance to the main sequence to better understand the underlying physical drivers of galaxies' integrated SED-averaged dust temperature.

\section{Sample and Observations}

\subsection{Sample Selection}

Seven unlensed, spectroscopically confirmed DSFGs were chosen from $400 \operatorname{arcmin}^{2}$ SCUBA-2 450 and $850 \mu \mathrm{m}$ maps of the inner COSMOS field (Casey et al. 2013). Of the 31 SCUBA-2-detected COSMOS sources with spectroscopic redshifts in Casey et al. (2017), we selected seven of the brightest sources (single-dish $S_{850}>2 \mathrm{mJy}$ ) to span a very broad range of dust SEDs from cold $(\sim 18 \mathrm{~K})$ to warm $(\sim 70 \mathrm{~K})$ based on their Herschel SPIRE and SCUBA-2 photometry. The DSFGs were selected at the time for having known spectroscopic redshifts of $z=1$ to 3 (Casey et al. 2017), and updated information on this sample gives a total spanned redshift range of $1.4<z<4.6$. The galaxy initially thought to have the coldest SED, 450.03 (also known as AzTEC2), was identified initially to have a spectroscopic redshift of 1.123 from an optical/infrared (OIR) counterpart (Casey et al. 2017) but is now confirmed to sit at $z=4.61$ from a serendipitous detection of [C II] in our ALMA data and concurrent confirmation via CO (5-4) line emission from NOEMA observations (JiménezAndrade et al. 2020). The ALMA centroid is offset from the OIR source by 1 ". 6 (see Casey et al. 2017 for details). The redshift for source 850.04 was originally estimated to be $z=1.436$, but closer inspection reveals a more likely redshift solution of $z=3.31_{-0.81}^{+0.76}$, which is a photometric redshift from the Laigle et al. (2016) COSMOS catalog. This photometric counterpart is 1 " 0 offset from the spectroscopically confirmed source at $z=1.436$ (Casey et al. 2017) but significantly closer $(0 . .3)$ from the centroid of ALMA emission.

\subsection{ALMA Data}

Observations of these seven DSFGs were carried out as part of the ALMA Cycle 3 program 2015.1.00568.S (PI: Casey). With a requested spatial resolution of 0 ." 1 , observations were made in both compact (C36-3) and extended (C36-6) configurations. The compact and extended configurations had maximum baselines of $462.9 \mathrm{~m}$ and $1.8 \mathrm{~km}$, respectively, with 36 antennas. The observations targeted dust continuum emission at the nominal band 7 frequency centered at $345 \mathrm{GHz}$ or $870 \mu \mathrm{m}$. At this frequency, ALMA has a 17!' 3 primary beam (FWHM), and we utilized the "single continuum" spectral mode.

We reduced and calibrated the raw data from the compact configuration to produce $u-v$ data products using the Common Astronomy Software Applications (CASA) version 4.5.3. During calibration, data from antennas with irregular amplitudes were flagged. To produce optimum images for total flux recovery, we imaged the compact-configuration data using the CLEAN algorithm of CASA with Briggs weighting and robust $=2$ (equivalent to natural weighting) and applied a primary beam correction. The resulting images have a resolution of 0 ". $68 \times 0$ ". 49 . The compact data set was used in calculating the flux densities, which are given in Table 1.

We created a higher-resolution set of maps by combining the raw data from the compact and extended configurations and then reducing and calibrating the data using the same method described above. We tested different robust values with the CLEAN algorithm and found the resulting size of each source to be consistent and independent of the robust parameter. Thus, we adopt a robust value of 1 for the full sample, as it maximizes the source signal-to-noise ratio for the most extended source in the sample (850.95) while maintaining a relatively high angular resolution. The resulting resolution is 0 " $24 \times 0$ " 27 . This does not meet the requested 0 " 12 resolution, but even with a robust value of 0 , the spatial resolution is still not as good as requested. The combined data were used to calculate the effective radii, which are also given in Table 1. Images of the sample are shown in Figure 1, and overlays with optical/nearinfrared data are shown in Figure 2 on the same scale.

\subsection{Biases in the Sample?}

The data sample used in this analysis does not fully sample the full parent population of galaxy dust SEDs but is representative of the breadth of DSFGs at high $z$. The COSMOS sources from Casey et al. (2017) were chosen explicitly to have the broadest range of SEDs and are selected at either $450 \mu \mathrm{m}$ or $850 \mu \mathrm{m}$, which together is unbiased with respect to SED dust temperatures in the range of 20-100 K (e.g., Casey et al. 2013; Roseboom et al. 2013). The ALESS sample selected at $870 \mu \mathrm{m}$ only is more likely to be biased toward colder dust temperatures (Chapman et al. 2004a; Casey et al. 2009), but we see a large range of temperatures well represented in the sample, similar to the COSMOS sample. Furthermore, while $870 \mu \mathrm{m}$ selection alone is intrinsically biased and favoring sources with colder SEDs, those that have wellsampled FIR SEDs (and high-resolution ALMA imaging) are not biased with respect to $\lambda_{\text {peak }}$ because detection in the Herschel bands bracketing the SED peak is implicitly required. In other words, many ALESS sources without Herschel SPIRE detections (i.e., those with cold SEDs) would not have well-sampled FIR SEDs, are unlikely to have received high-resolution ALMA follow-up, and thus are excluded from our analysis here.

The sample (both COSMOS and ALESS DSFGs) could be seen as biased toward galaxies with high SFRs, but sources with high SFRs are the only ones for which the high spatial resolution measurements made in this paper are possible with a reasonable investment in ALMA time. This has potential impact on our ability to probe the relationship between $\lambda_{\text {peak }}$ and $L_{\mathrm{IR}}$ in a fully unbiased way, given the limited dynamic range compared to galaxy populations at large. This is discussed more fully in Section 5.2.2. The combined sample does sample galaxies' relative distance from the main sequence well, in line with the expected dynamic range of much larger populations of studied galaxies. They span two orders of magnitude in sSFR from $\sim 0.6$ to $60 \mathrm{Gyr}^{-1}$, and about one order of magnitude in orthogonal distance from the main sequence; more methods to quantify galaxies' distance to the main sequence are discussed later in Section 4.4.

The primary goal of this work is to assess the relative strength of correlation between luminosity-weighted dust temperature (as measured observationally through the proxy $\left.\lambda_{\text {peak }}\right)$ and other physical characteristics of high- $z$ galaxies, including quantities constrained by the FIR-emitting region as 
Table 1

Redshifts, Total Flux Densities, Half-light Radii as Measured by IMFIT at $870 \mu \mathrm{m}$, Rest-frame SED Peak Wavelengths, Infrared Luminosities, Star Formation Rates, and Stellar Masses for Our COSMOS SCUBA-2 and ALESS Sources

\begin{tabular}{|c|c|c|c|c|c|c|c|}
\hline Name & $z$ & $\begin{array}{l}S_{870} \\
(\mathrm{mJy})\end{array}$ & $\begin{array}{c}R_{\mathrm{e}} \\
(\mathrm{kpc})\end{array}$ & $\begin{array}{l}\lambda_{\text {peak }} \\
(\mu \mathrm{m})\end{array}$ & $\begin{array}{c}L_{\mathrm{IR}} \\
\left(L_{\odot}\right)\end{array}$ & $\begin{array}{c}\text { SFR } \\
\left(M_{\odot} \mathrm{yr}^{-1}\right)\end{array}$ & $\begin{array}{c}M_{\star} \\
\left(M_{\odot}\right)\end{array}$ \\
\hline 450.03 & $4.629^{\mathrm{a}}$ & $19.12 \pm 0.16$ & $1.35^{\mathrm{b}} \pm 0.06$ & $71.4 \pm 7.8$ & $(3.28 \pm 0.68) \times 10^{13}$ & $4900 \pm 1000$ & $\ldots^{\mathrm{c}}$ \\
\hline 850.04 & 3.31 & $13.67 \pm 0.12$ & $1.94 \pm 0.12$ & $98 \pm 9$ & $(8.9 \pm 1.2) \times 10^{12}$ & $1330 \pm 180$ & $(2.2 \pm 0.8) \times 10^{10}$ \\
\hline 450.14 & 1.523 & $2.05 \pm 0.03$ & $2.8 \pm 0.4$ & $130 \pm 30$ & $(1.2 \pm 0.7) \times 10^{12}$ & $190 \pm 100$ & $\ldots^{\mathrm{c}}$ \\
\hline 850.60 & 1.457 & $2.21 \pm 0.04$ & $1.81 \pm 0.28$ & $75 \pm 9$ & $(1.9 \pm 0.4) \times 10^{12}$ & $290 \pm 60$ & $\left(2.1_{-0.2}^{+0.1}\right) \times 10^{11}$ \\
\hline 850.95 & 1.556 & $1.21 \pm 0.03$ & $6.1 \pm 1.4$ & $98 \pm 22$ & $(1.2 \pm 0.5) \times 10^{12}$ & $180 \pm 80$ & $\left(3.8_{-1.0}^{+0.4}\right) \times 10^{10}$ \\
\hline 850.25 & 2.438 & $5.76 \pm 0.05$ & $2.76 \pm 0.22$ & $117 \pm 20$ & $(3.1 \pm 1.1) \times 10^{12}$ & $460 \pm 170$ & $\left(2.8_{-0.4}^{+0.1}\right) \times 10^{11}$ \\
\hline 450.09 & 2.472 & $6.07 \pm 0.07$ & $1.04 \pm 0.07$ & $71 \pm 10$ & $(8.3 \pm 1.5) \times 10^{12}$ & $1240 \pm 230$ & $\left(2.2_{-0.4}^{+0.1}\right) \times 10^{11}$ \\
\hline ALESS 3.1 & 4.24 & $8.3 \pm 0.4$ & $1.30 \pm 0.05$ & $69 \pm 9$ & $(1.64 \pm 0.25) \times 10^{13}$ & $2460 \pm 380$ & $\left(2.3_{-1.6}^{+1.0}\right) \times 10^{11}$ \\
\hline ALESS 5.1 & 2.86 & $7.8 \pm 0.7$ & $2.00 \pm 0.10$ & $106 \pm 12$ & $(4.9 \pm 1.0) \times 10^{12}$ & $730 \pm 150$ & $\left(2.9_{-0.8}^{+0.2}\right) \times 10^{11}$ \\
\hline ALESS 9.1 & 4.50 & $8.8 \pm 0.5$ & $1.50 \pm 0.05$ & $71 \pm 9$ & $(1.63 \pm 0.26) \times 10^{13}$ & $2430 \pm 390$ & $\left(5.6_{-2.7}^{+1.3}\right) \times 10^{11}$ \\
\hline ALESS 10.1 & 0.76 & $5.3 \pm 0.5$ & $2.60 \pm 0.20$ & $190 \pm 9$ & $(2.3 \pm 0.4) \times 10^{11}$ & $34.5 \pm 5.5$ & $(1.2 \pm 0.3) \times 10^{10}$ \\
\hline ALESS 15.1 & 1.93 & $9.0 \pm 0.4$ & $2.40 \pm 0.10$ & $125 \pm 5$ & $(2.95 \pm 0.24) \times 10^{12}$ & $440 \pm 40$ & $\left(3.1_{-1.8}^{+1.3}\right) \times 10^{11}$ \\
\hline ALESS 17.1 & 1.54 & $8.4 \pm 0.5$ & $1.75 \pm 0.05$ & $138 \pm 5$ & $(1.88 \pm 0.18) \times 10^{12}$ & $281 \pm 27$ & $\left(2.3_{-1.7}^{+0.1}\right) \times 10^{11}$ \\
\hline ALESS 29.1 & 1.44 & $5.9 \pm 0.4$ & $1.35 \pm 0.05$ & $136 \pm 12$ & $(1.37 \pm 0.23) \times 10^{12}$ & $200 \pm 30$ & $\left(3.0_{-2.1}^{+1.2}\right) \times 10^{11}$ \\
\hline ALESS 39.1 & 2.44 & $4.3 \pm 0.3$ & $1.95 \pm 0.10$ & $100 \pm 7$ & $(3.3 \pm 0.4) \times 10^{12}$ & $490 \pm 70$ & $\left(4.5_{-1.6}^{+1.0}\right) \times 10^{10}$ \\
\hline ALESS 45.1 & 2.34 & $6.0 \pm 0.5$ & $2.15 \pm 0.15$ & $117 \pm 10$ & $(2.7 \pm 0.5) \times 10^{12}$ & $400 \pm 70$ & $\left(5.1_{-2.3}^{+1.5}\right) \times 10^{11}$ \\
\hline ALESS 67.1 & 2.12 & $4.5 \pm 0.4$ & $1.85 \pm 0.15$ & $72.2 \pm 2.6$ & $(9.4 \pm 0.9) \times 10^{12}$ & $1400 \pm 140$ & $\left(2.4_{-2.1}^{+2.4}\right) \times 10^{11}$ \\
\hline ALESS 112.1 & 2.32 & $7.6 \pm 0.5$ & $1.90 \pm 0.10$ & $109 \pm 6$ & $(4.1 \pm 0.5) \times 10^{12}$ & $610 \pm 70$ & $\left(1.0_{-0.5}^{+0.4}\right) \times 10^{11}$ \\
\hline
\end{tabular}

Notes. Measurements of peak wavelengths, infrared luminosities, and SFRs are done by the same method for both the COSMOS sample and the 11 ALESS sources we analyze. Measurements for 450.03 are for the total circularized size and total $L_{\mathrm{IR}}$ of both components. ALESS flux densities are from Swinbank et al. (2014), and sizes are from Hodge et al. (2016), derived in a fully consistent manner as for our COSMOS sample.

${ }^{\mathrm{a}}$ The precision quoted on the redshift indicates whether or not the source has a spectroscopic redshift (four significant figures) or a photometric redshift (three significant figures).

${ }^{\mathrm{b}}$ Total circularized size of both components of 450.03 . The individual half-light radii measured by IMFIT are $1.24 \mathrm{kpc}$ for the brightest source and $1.08 \mathrm{kpc}$ for the secondary source.

${ }^{c}$ Stellar masses are not provided for 450.03 or 450.14 . In the case of 450.03 , this is due to significant blending with several foreground galaxies on angular scales that precludes measurement of near-infrared Spitzer flux densities; in the case of 450.14, there is a superposition of two galaxies of different redshifts within a $2^{\prime \prime}$ region, making a proper separation of the background/foreground stellar mass distribution impossible. Both cases are discussed at greater length in Casey et al. (2017).

measured by high-resolution ALMA dust continuum imaging. Thus, this set of observations constitutes a well-constructed and relatively unbiased sample for our purposes.

\section{Calculations and Results}

\subsection{ALMA $870 \mu m$ Flux Densities}

Total integrated flux densities were measured directly from the compact ALMA data using a method similar to the one described in Hodge et al. (2016). We converted the maps from Jy beam ${ }^{-1}$ to mJy pixel ${ }^{-1}$ and masked emission less than $2 \sigma$. We then summed contiguous emission within an aperture of radius $3 \times b_{\text {maj }}$, where $b_{\text {maj }}$ is the FWHM (major axis) of the synthesized beam. The resulting total band 7 flux densities are given in Table 1. The median flux density of our seven sources is $5.8 \pm 3.7 \mathrm{mJy}$ at $870 \mu \mathrm{m}$.

In general, when measuring the flux density, only contiguous emission was summed, with one exception. Source 450.03 is composed of two sources separated by $3^{\prime \prime}$ at the same redshift (Jiménez-Andrade et al. 2020), both of which were included in the total reported flux density.

To check our flux density calculations, we used the IMFIT tool in CASA and found that our calculations agree with the IMFITderived densities for all but one of our sources. Source 850.95 differed with less than $2 \sigma$ significance of deviation. The average ratio between IMFIT and aperture flux density measurements was $0.96 \pm 0.10$.
In addition, we compare the flux densities as measured by ALMA to the single-dish flux densities obtained for the COSMOS sources from SCUBA-2 at $850 \mu \mathrm{m}$ using the singledish JCMT and for the ALESS sources from LABOCA at $870 \mu \mathrm{m}$. The average ratio of ALMA to single-dish flux densities was $0.94 \pm 0.21$, as shown in Figure 3 . Note that the expected ratio of $850 \mu \mathrm{m}$ to $870 \mu \mathrm{m}$ flux is $\approx 0.9$ for RJ tail emission.

\subsection{Measuring Dust Sizes and Morphology}

We quantitatively examine the size of the $870 \mu \mathrm{m}$ dust emission by fitting each source in the image plane with twodimensional Sérsic and Gaussian models. The Sérsic model was applied using GALFIT, and the Gaussian model was applied using CASA IMFIT.

Dust emission half-light effective radii $\left(R_{\mathrm{e}}\right)$ were measured using GALFIT on the ALMA maps in the image plane. GALFIT is a 2D fitting tool that models the profiles of astronomical objects using parametric functions (Peng et al. 2010). Here we use GALFIT on ALMA data to test for non-Gaussian source morphologies, which is most easily applied in the image plane. The full width at half maximum (FWHM) was measured for each source using CASA IMFIT, which is also applied in the image plane. Most of our sources are moderately resolved-more than two beams across in size-so size measurements are unlikely to be affected by fitting methods. Thus, making measurements in the image plane rather than the $u$-v plane is justified. 


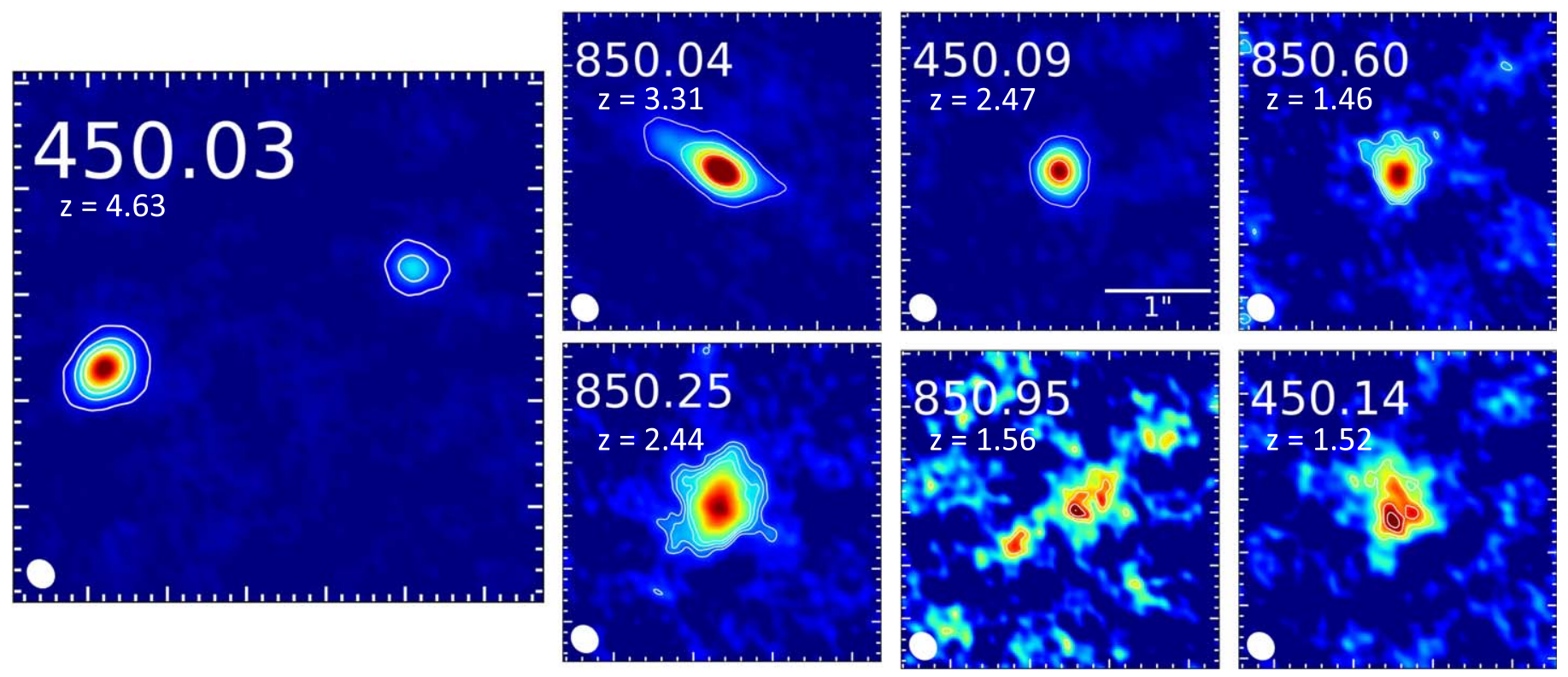

Figure 1. ALMA $870 \mu \mathrm{m}$ images, proportional to the dust mass distribution for all sources except for perhaps 450.03 , which sits at a much higher $z$. All images are $3^{\prime \prime} \times 3^{\prime \prime}$, except for 450.03 , which is $5^{\prime \prime} \times 5^{\prime \prime}$ to show its two components (Jiménez-Andrade et al. 2020). The ALMA beam is shown in the lower left corner. For sources $450.03,850.04$, and 450.09 , the contours start at $5 \sigma$ and increase by $10 \sigma$. For the remaining sources, the contours start at $3 \sigma$ and increase by $1 \sigma$.
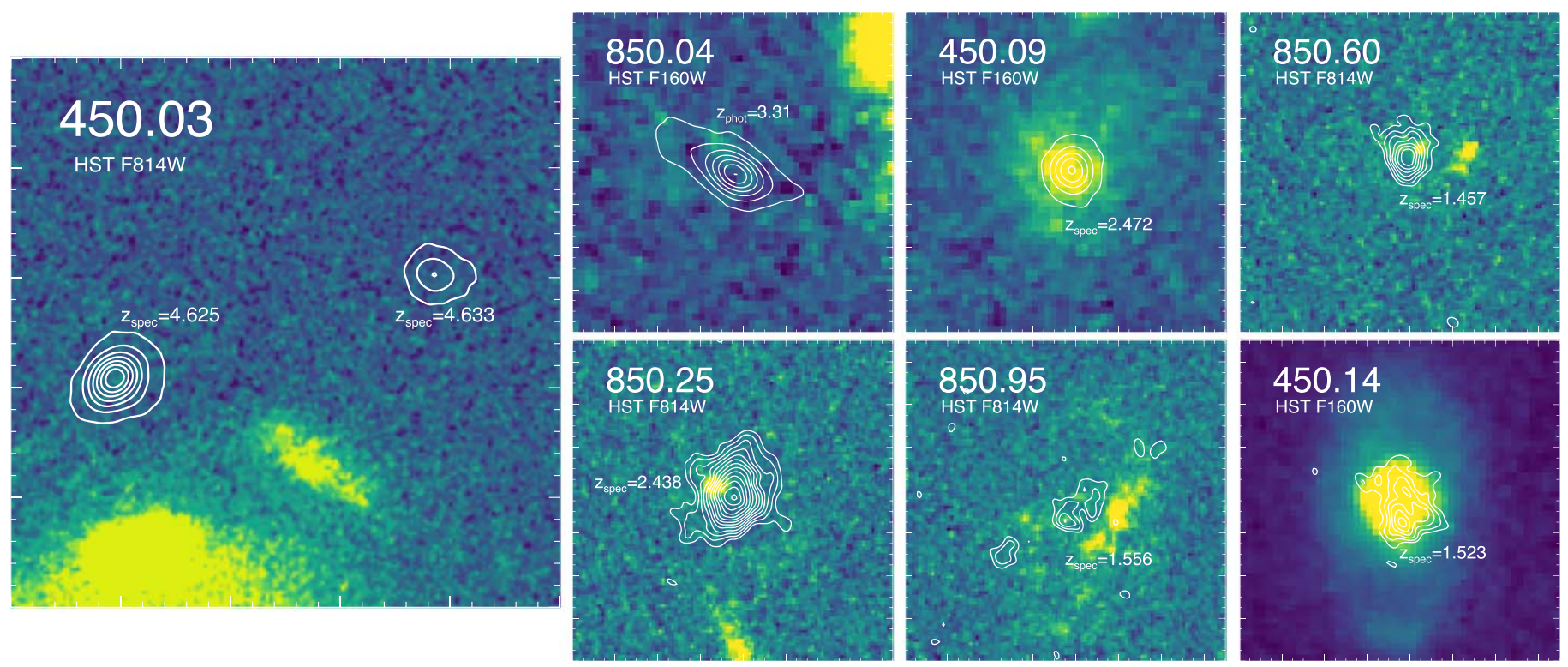

Figure 2. Optical or near-infrared imaging from Hubble Space Telescope of the COSMOS sample, with ALMA contour levels and the same scale as in Figure 1. Where near-infrared imaging exists (from the CANDELS survey), it is shown (in the WFC3/F160W filter); otherwise, $i$-band imaging from ACS/F814W is shown. All sources here have secure spectroscopic redshifts (from either the millimeter or near-infrared) except 850.04, whose photometric redshift of $z=3.31$ is based on an OIR counterpart separated only by $0 . " 4$ from the measured centroid of dust emission. The ALESS sample has presented similar cutouts against HST imaging in Chen et al. (2015).

To address whether the weighting applied with the CLEAN algorithm has an impact on size measurement in the image plane, measurements were also made in the $u-v$ plane using CASA UVMODELFIT to fit a Gaussian model. The UVMODELFIT and IMFIT measurements agree within uncertainties for four of our sources. There is a discrepancy between $u$ - $v$ and imageplane sizes for 450.03 and 850.25 , which we attribute to significant excess emission from the core of the systems, which is evident in the IMFIT residual images. There was also a discrepancy in the measurements for source 850.95 , which we attribute to the IMFIT model extending beyond the region of significant flux associated with the source. In general, the measured Gaussian FWHMs in the $u$ - $v$ plane are systematically offset from the FWHMs in the image plane measured by IMFIT such that the median ratio of $u$-v-plane to image-plane FWHMs is $0.81 \pm 0.08$. Our scientific results rely on relative size comparisons of objects within the sample (and extended to the ALESS sample) rather than absolute sizes. The results of GALFIT are shown in Figure 4, along with the residuals and models. It is a known problem that errors from GALFIT are underestimated; therefore, we used the IMFIT results in our analysis and report IMFIT sizes in Table 1. 


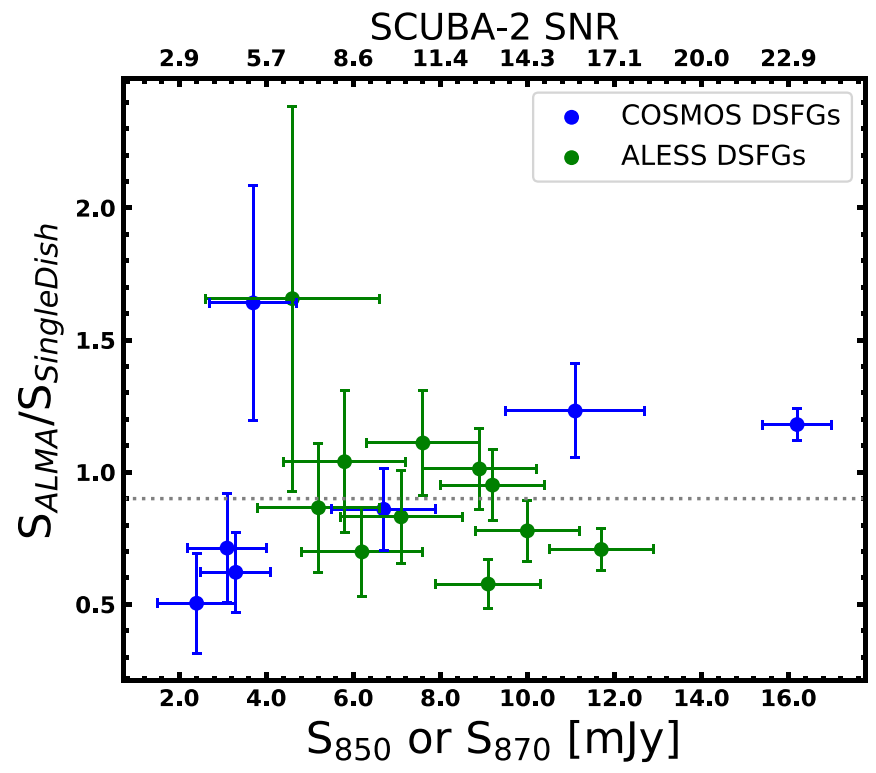

Figure 3. Single-dish flux $\left(S_{850}\right.$ or $\left.S_{870}\right)$ vs. ratio of ALMA to single-dish flux $\left(S_{\text {ALMA }} / S_{\text {SingleDish }}\right)$ for each galaxy. Single-dish fluxes are SCUBA-2 $(850 \mu \mathrm{m})$ for COSMOS sources and LABOCA $(870 \mu \mathrm{m})$ for ALESS sources. The SCUBA-2 signal-to-noise ratio was calculated using $\sigma=0.7 \mathrm{mJy}^{\text {beam }}{ }^{-1}$. The dashed horizontal line at $S_{\text {ALMA }} / S_{\text {SingleDish }}=0.9$ indicates the expected flux ratio on the RJ tail of a blackbody. Three COSMOS sources $(450.09,450.03$, and 850.04) have higher ALMA flux densities than SCUBA-2 flux densities, and the remaining COSMOS sources have lower ALMA flux densities. Four ALESS sources (15.1, 17.1, 45.1, and 112.1) have higher ALMA flux densities than LABOCA flux densities, and the remaining ALESS sources have lower ALMA flux densities.

The sizes measured here are the mass-weighted sizesmeasured on the RJ tail of the SED-rather than the luminosity-weighted sizes, which we would measure closer to the peak of the SED. The only exceptions to this are those galaxies that sit at $z>3$ in our sample (5 of 18); at these redshifts, observed $870 \mu \mathrm{m}$ probes rest-frame wavelengths shortward of $200 \mu \mathrm{m}$, which may be more prone to tracing an optically thick regime of the SED in some cases (dependent on the actual dust column). We explore the impact of these higher-redshift sources on our final results later in Section 5. A dearth of data in the literature makes it difficult to know whether measured size varies substantially along the RJ tail, but the limited one-off measurements that do exist suggest that it is safe to assume that any measurement on the tail should be consistent within measurement uncertainties. In addition, these sizes are distinct from the sizes of the galaxies traced by stellar emission; the two are often spatially distinct in DSFGs, such that the rest-frame UV/optical emission is heavily obscured by dust and thus does not serve as an adequate probe of the characteristic scale of the ISM (Biggs \& Ivison 2008; Chen et al. 2015; Hodge et al. 2015, 2016; Lang et al. 2019). It should also be noted that dust continuum sizes are systematically smaller and not entirely consistent with radio continuum sizes (which probe synchrotron emission scattering off of supernova remnants), the measurements of which exist for much larger samples than those with dust continuum size measurements (Miettinen et al. 2017).

The effective radii measured by GALFIT range from 0 ". 13 to 0 ". 69 with a median of $R_{\mathrm{e}}=0$ "' $32 \pm 0$ ". 09 . Using the redshift of each source, we convert these angular sizes to physical sizes, which range from 1.1 to $5.9 \mathrm{kpc}$ (median $R_{\mathrm{e}}=2.7 \pm 0.8 \mathrm{kpc}$ ).
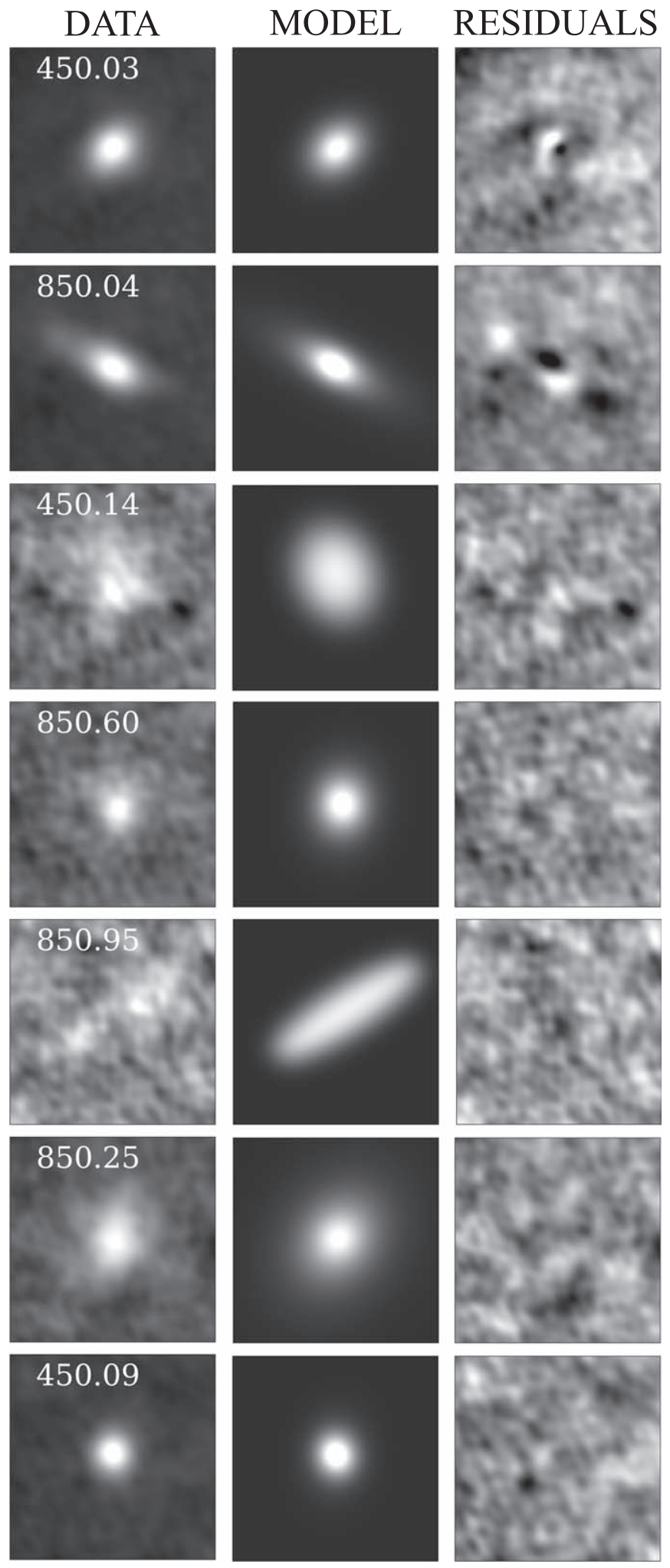

Figure 4. $2^{\prime \prime} \times 2^{\prime \prime}$ model and residual images generated by GALFIT for each source. Images on the far left are the original ALMA $870 \mu \mathrm{m}$ images with robust $=1$. Only the brightest component of 450.03 is shown.

Source 850.95 was an outlier at $5.9 \pm 3.3 \mathrm{kpc}$. It is known to be somewhat abnormally large as also traced by $\mathrm{H} \alpha$ kinematics (Drew et al. 2018). Source 850.95 also has the lowest signal-tonoise ratio among our sources. The remaining sources ranged from 1.1 to $3.2 \mathrm{kpc}$ (median $R_{\mathrm{e}}=2.3 \pm 0.5 \mathrm{kpc}$ ). 
The FWHMs (deconvolved from the restoring beam) measured by IMFIT are shown in Table 1. They range from 0 " 26 to $1 . " 45$, which convert to $2.1-12.3 \mathrm{kpc}$ on physical scales. The half-light radii quoted in Table 1 are equivalent to these FWHM measurements divided by two. The median Gaussian profile has an FWHM $=3.9 \pm 1.6 \mathrm{kpc}$. Excluding 850.95 , the median is $3.8 \pm 1.4 \mathrm{kpc}$.

The Gaussian profiles of the ALESS sources included in our analysis were measured via the same technique used here (Hodge et al. 2016), where the median FWHM $=3.8 \pm 0.5 \mathrm{kpc}$. The median size of our sources agrees with that of the ALESS sources, despite the fact that the ALESS sample is brighter than all but two of our sources. Overall, the DSFG populations from which the ALESS and COSMOS SCUBA-2 DSFGs are drawn are very similar, with the exception of the ALESS median total $870 \mu \mathrm{m}$ flux densities being $1.3 \times$ brighter. The median of our sources' half-light radii is also consistent within uncertainty with that found by Spilker et al. (2016) and Gullberg et al. (2019).

The Sérsic indices of our sources range from $n=0.3$ to 2.1, with a median of $n=0.8 \pm 0.4$. Our median $n$ is consistent with that of the ALESS sources in Hodge et al. (2016), whose median Sérsic index $n=0.9 \pm 0.2$ implies a non-Gaussian, exponential disk morphology. Gullberg et al. (2019) also found an exponential disk emission profile for a sample of DSFGs.

\subsection{SED Integrated Dust Temperature}

Wien's law states that $\lambda_{\text {peak }}=b / T_{\mathrm{d}}$ (where $b=2.898 \times 10^{-3}$ $\mathrm{mK}$ ) for an idealized blackbody, but this relationship deviates depending on the adopted opacity model of the blackbody as shown in Figure 20 of Casey et al. (2014). Thus, we parameterize the luminosity-weighted dust temperature $\left(T_{\mathrm{d}}\right)$ instead as the observable $\lambda_{\text {peak }}$, the rest-frame wavelength where the dust SED peaks. The observable $\lambda_{\text {peak }}$ is not sensitive to opacity assumptions and is a better trace of the observed peak in the SED in any observational sample without high-quality constraints on the underlying dust opacity.

SEDs in this work were fitted using the technique described in Casey (2012), based on a single dust temperature modified blackbody fit with a mid-infrared power law shortward of $\sim 70 \mu \mathrm{m}$ in the rest frame. The mid-infrared power law represents a superposition of modified blackbodies at warm to hot temperatures, where the least amount of dust mass is heated to the hottest temperatures, and the vast majority of the mass of dust in the ISM is at much colder temperatures, represented by the temperature of the fit at rest-frame wavelengths $\gtrsim 200 \mu \mathrm{m}$. Because our sample has somewhat limited rest-frame mid-infrared photometric constraints, we fix the mid-infrared power-law slope to $\alpha_{\mathrm{MIR}}=2$, representative of the average measured power-law slope for local LIRGs and ULIRGs that have well-sampled midIR SEDs (e.g., U et al. 2012).

The photometric points used in the fitting include ALMA $(870 \mu \mathrm{m})$, Herschel SPIRE $(250,350,500 \mu \mathrm{m})$, SCUBA-2 $(450,850 \mu \mathrm{m})$, and Spitzer $(24 \mu \mathrm{m})$. Points are weighted relative to their signal-to-noise ratio, with the exception of the Spitzer $24 \mu \mathrm{m}$ point, whose signal-to-noise ratio is capped at 10 for the purposes of these bulk SED fits. The $24 \mu \mathrm{m}$ photometry often is a very high signal-to-noise ratio measurement but is not always in agreement with the underlying SED model for dust continuum emission alone, due to the possible contribution of PAH emission features and silicate absorption in the rest-frame mid-infrared but not directly modeled here. We also note that use of the response function of the various FIR/millimeter passbands is not necessary using the Casey (2012) fitting method given the lack of $\approx 1 \%$ precision in the photometry in the FIR regime (see also Casey 2020).

While the bulk SED, including the measured IR luminosity or rest-frame peak wavelength, is not impacted by the adopted opacity model, here we adopt a fixed opacity model for all SEDs such that $\tau=1$ at $200 \mu \mathrm{m}$ following Conley et al. (2011). Given that our observations include spatially resolved dust maps, we can test whether this assumption is appropriate for this sample by inferring the wavelengths at which $\tau=1$ from the dust column density. Using the total $870 \mu \mathrm{m}$ flux densities from Section 4.1, sizes from Section 4.2, a fixed mass-weighted dust temperature of $25 \mathrm{~K}$, and dust mass absorption coefficients from Li \& Draine (2001), we infer that the wavelength at which the SEDs become optically thick varies from 10 to $300 \mu \mathrm{m}$ (with an average $\lambda(\tau=1)=80 \pm 50 \mu \mathrm{m})$. Given the uncertainties in the derivation of dust mass itself, we choose to apply the uniform assumption to our SEDs rather than tuning each fit according to its measured dust column. The SEDs are shown in Figure 5, and the measured peak wavelengths and IR luminosities are also given in Table 1 . We also refit SEDs for the ALESS sources described in Hodge et al. (2016), whose multiwavelength photometry is given in Swinbank et al. (2014). Fitting SEDs for both the COSMOS SCUBA-2 DSFGs and the ALESS sources was done in a fully consistent manner.

\subsection{Star Formation Rates and Stellar Masses}

SFRs were calculated using the total infrared luminosities derived from the SED fits in Section 4.3 and the logarithmic constant of conversion, $\log \left(C_{\mathrm{IR}}\right)=43.41$ from Kennicutt \& Evans (2012). We do not have reliable stellar mass estimates for 450.03 and 450.14 owing to foreground-background source contamination (see Casey et al. 2017), so they are omitted from all analysis requiring measurements of $M_{\star}$. The stellar masses of the COSMOS sample are presented in Casey et al. (2017), and for the ALESS sources they are presented in da Cunha et al. (2015). All stellar masses used in this study are derived with MAGPHYS (da Cunha et al. 2008, 2015) in a uniform fashion using stellar population models from Bruzual \& Charlot (2003). For the two sources for which the redshifts were corrected, we reran the MAGPHYS modeling accordingly. Note that the stellar masses of DSFGs are often highly uncertain, though not necessarily systematically so, as they can also be overestimated by applying an inappropriate star formation history (see discussions in Hainline et al. 2011; Michałowski et al. 2012). The uncertainties on the stellar masses are consistent with the broad uncertainties on DSFG stellar masses in general. We note that our MAGPHYS fits are used exclusively to derive stellar masses, and the MAGPHYS fits are not used in this work to infer FIR SED characteristics like dust temperature or SFR; this is because of the potential for the rest-frame optical/near-infrared SED to be highly decoupled from FIR emission in DSFGs, as well as our desire to directly test the FIR fits alone and independently of the rest-frame optical/nearinfrared emission used to measure the stellar masses. We also calculated the sSFR, where $\mathrm{sSFR}=\mathrm{SFR} / M_{\star}$.

Figure 6 shows the COSMOS and ALESS sources in relation to the SFR $-M_{\star}$ relation, also called the galaxy main sequence. In red, we show the main sequence of star-forming galaxies with a quadratic function of the form used in Whitaker et al. (2014) applied to COSMOS data $(1.5 \leqslant z \leqslant 2.5)$ from Laigle et al. (2016). We applied a Markov Chain Monte Carlo (MCMC) approach to derive the parameters of the fit for the 


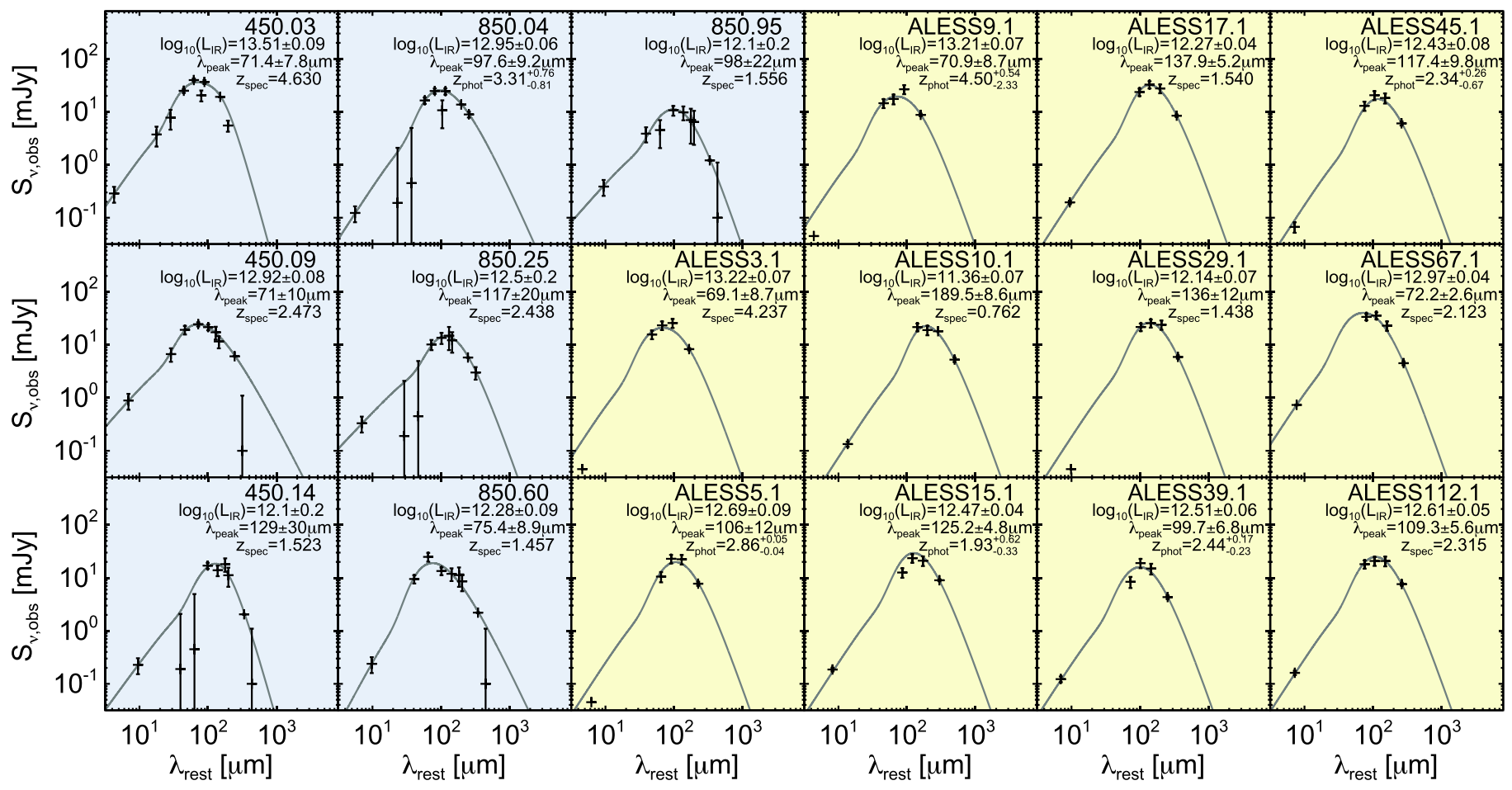

Figure 5. Infrared SEDs for our sample and the ALESS sample included in our analysis. The photometry is as reported in Casey et al. (2013, 2017), Swinbank et al. (2014), and Hodge et al. (2016), and SEDs are fit using the single modified blackbody plus mid-infrared power-law technique detailed in Casey (2012). We tested different permutations of SED fits (fixing or allowing variance in the mid-infrared power-law slope, the emissivity spectral index, or assumed opacity of the dust near the peak) and find that measurements of $L_{\mathbb{R}}$ or $\lambda_{\text {peak }}$ are insensitive to higher-order permutations within the margin of error. Noted in each panel is the source redshift, $L_{\mathbb{I R}}$, and $\lambda_{\text {peak }}$ as also stated in Table 1 .

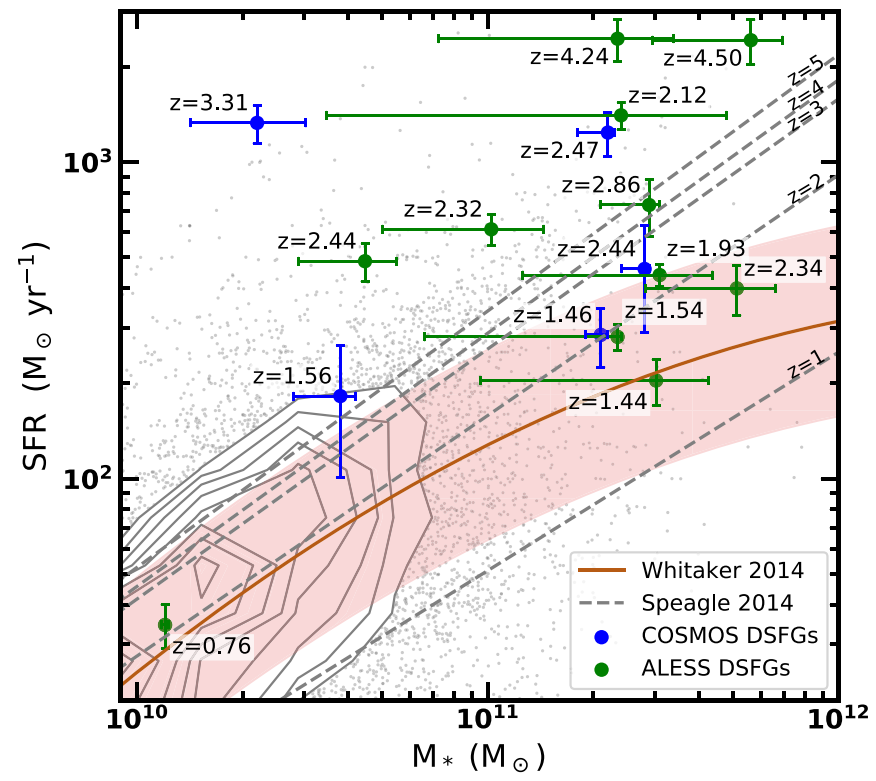

Figure 6. Five of our COSMOS sources (blue) and 11 of the ALESS sources (green) overplotted on the galaxy main sequence. Two of our COSMOS sources (450.03 and 450.14) are not included here because we do not have reliable stellar mass estimates for them. The orange line is the best-fit polynomial of the form used in Whitaker et al. (2014) at $z=2$. Note that there is significant scatter in the main sequence, and the average scatter is represented here as the red shaded region, with a width of 0.3 dex. Sources in the red shaded region or with error bars extending into the red shaded region lie on the main sequence. The dashed lines are the cosmic time-dependent best fits from Speagle et al. (2014) for redshifts $z=1,2,3$, 4, and 5 .

broader COSMOS data set. Also shown are the cosmic timedependent best fits from Speagle et al. (2014) for redshifts $z=1,2,3,4$, and 5 .
For each galaxy in our sample, we also compute a parameterized "distance" to the main sequence, or $D_{\mathrm{MS}}$. $D_{\mathrm{MS}}$ represents the orthogonal distance between the main sequence at the source's redshift and its measured value in the $\log M_{\star}-\log$ SFR plane. We compute orthogonal distances to the main sequence rather than projected distances in SFR or sSFR to minimize the impact of measurement uncertainties in both $M_{\star}$ and SFR for each galaxy. The main sequence used to calculate $D_{\mathrm{MS}}$ for each source were the cosmic time-dependent curves given in Speagle et al. (2014); however, we note that computing $D_{\mathrm{MS}}$ using a quadratic form of the main sequence as in Whitaker et al. (2014) produces similar results, ultimately not changing the conclusions of our work no matter which definition of $D_{\mathrm{MS}}$ is adopted. Similarly, as we discuss later in Section 5, we test for correlation between $\lambda_{\text {peak }}$ and $\Delta \mathrm{sSFR}$, finding results that are consistent with our adopted $D_{\mathrm{MS}}$.

\section{Discussion}

\subsection{Expected Relationship of Dust Geometry to Dust Temperature}

Most galaxies for which dust temperatures have been constrained lack spatially resolved measurements needed to investigate the effects of dust geometry on dust temperature. Thus, the underlying physical driver of a galaxy's globally averaged dust temperature has not been directly constrained. Before ALMA, there were small samples of high- $z$ galaxies that had resolved radio or millimeter sizes (Chapman et al. 2004b; Biggs \& Ivison 2008; Casey et al. 2009; Younger et al. 2009), but the observations needed to determine accurate sizes for larger samples of DSFGs require the order-of-magnitude improvements in sensitivity brought by ALMA (see Hodge et al. 2016; Rujopakarn et al. 2016; Simpson et al. 2017; Elbaz et al. 2018; Gullberg et al. 2019). In this paper, we have 
presented a sample of DSFGs with both high-resolution dust size constraints and good dust SED constraints, allowing a more thorough investigation into the physical drivers of galaxies' dust SEDs.

This study is theoretically motivated by the application of the Stefan-Boltzmann law on galaxy scales. Specifically, it relates the emergent IR luminosity to the effective size of the blackbody $\left(R_{\mathrm{e}}\right)$ and temperature $\left(T_{\mathrm{d}}\right)$ of an optically thick spherical blackbody:

$$
L_{\mathrm{IR}}=4 \pi R_{\mathrm{e}}^{2} \sigma T^{4} .
$$

The Stefan-Boltzmann constant is $\sigma=5.670 \times 10^{-5} \mathrm{erg} \mathrm{s}^{-1}$ $\mathrm{cm}^{-2} \mathrm{~K}^{-4}$. Galaxies are certainly not as simple as stars in the application of such a relation. Nevertheless, approximating galaxies' IR emission as an optically thick shell of dust surrounding an obscured point source (i.e., deeply embedded OB associations in star-forming regions) would result in the following expected relation between the dust's temperature, IR luminosity, and dust shell radius:

$$
\log \Sigma_{\mathrm{IR}}=\log (4 \sigma)+4 \log \left(T_{\text {dust }}\right),
$$

where $\Sigma_{\mathrm{IR}}$ is the IR luminosity surface density $\left(=L_{\mathrm{IR}} / \pi R_{\mathrm{e}}^{2}\right)$. Of course, galaxies' geometry is unlikely to be well represented as a spherical shell, and the surface area of the emergent IR flux does indeed play a role in the expected relation. If instead of a spherical shell we assume some uniform planar distribution of UV emission underneath an optically thick screen (as in a galaxy disk, with half-light radius $R_{e}$ ), we would expect the following relationship instead:

$$
\log \Sigma_{\mathrm{IR}}=\log (\sigma)+4 \log \left(T_{\text {dust }}\right),
$$

which is a factor of four different than the spherical shell: one factor of two given the modified surface area of a disk as $2 \pi R_{\mathrm{e}}^{2}$, and one factor of two accounting for $R_{e}^{2}$ being a measured halflight radius. Then, given our use of optically thick SEDs (described in Section 4.3), the relationship between dust temperature and $\lambda_{\text {peak }}$ can be approximated as

$$
\log \left(T_{\text {dust }} / \mathrm{K}\right) \approx 3.756-1.048 \log \left(\lambda_{\text {peak }} / \mu \mathrm{m}\right)
$$

in the $15-70 \mathrm{~K}$ range. ${ }^{9}$ This gives a predicted relationship between $\lambda_{\text {peak }}$ and $\Sigma_{\mathrm{IR}}$ of

$$
\log \left(\Sigma_{\mathrm{IR}} /\left[L_{\odot} \mathrm{kpc}^{-2}\right]\right) \approx 20.18-4.19 \log \left(\lambda_{\text {peak }} / \mu \mathrm{m}\right) .
$$

Note that the expectation using Wien's law directly instead of the approximation given in Equation (4) would yield

$$
\begin{aligned}
\log \left(\sum_{\mathrm{IR}} /\left[L_{\odot} \mathrm{kpc}^{-2}\right]\right)= & \log \left(\sigma b^{4}\right)-4 \log \left(\lambda_{\text {peak }} / \mu \mathrm{m}\right) \\
& \approx 19.00-4 \log \left(\lambda_{\text {peak }} / \mu \mathrm{m}\right),
\end{aligned}
$$

where $b=2.898 \times 10^{3} \mu \mathrm{m} \mathrm{K}$.

One key caveat here is that the Stefan-Boltzmann law should only be applicable to optically thick blackbodies, and the dust in galaxies' ISM is often far from optically thick at all wavelengths (of course, it is a huge benefit that ISM dust is often optically thin on the RJ tail of blackbody emission, allowing for a direct measurement of galaxies' dust mass from measured flux density). We return to the conundrum of dust

\footnotetext{
9 Note that this only deviates by 5\% from Wien's law for optically thin, idealized blackbodies.
}

opacity again in Section 5.2.1, where we derive the relationship between $\Sigma_{\mathrm{IR}}$ and $\lambda_{\text {peak }}$ empirically.

\subsection{What Correlates Best with Dust Temperature?}

Here we investigate the relative strengths of correlations between $\lambda_{\text {peak }}$ (our observational proxy for dust temperature) and each of the following physical properties of galaxies: sSFR, $D_{\mathrm{MS}}$, SFR, and $\Sigma_{\mathrm{IR}}$. All of these quantities have been argued to correlate tightly with galaxies' dust temperatures, for example, sSFR and $D_{\mathrm{MS}}$ (e.g., Magnelli et al. 2014), SFR or $L_{\mathrm{IR}}$ (e.g., Chapman et al. 2003b; Symeonidis et al. 2013), and $\Sigma_{\mathrm{IR}}$ (e.g., Chanial et al. 2007; Lutz et al. 2016). For example, Magnelli et al. (2014) report that $T_{\mathrm{d}}$ correlates more strongly with SSFR and parameterized distance to the main sequence $\left(D_{\mathrm{MS}}\right)$ than $L_{\mathrm{IR}}$ (they lacked direct measures of $R_{\mathrm{e}}$ and so could not investigate $L_{\mathrm{IR}} R_{\mathrm{e}}^{-2}$ ). In addition, they deduced that cold galaxies $(\lesssim 25 \mathrm{~K})$ sit on the main sequence and warm galaxies $(\gtrsim 30-80 \mathrm{~K})$ lie above the main sequence (Magnelli et al. 2014).

Here we use this high- $z$ sample with well-measured sizes and SEDs to directly constrain which of these physical correlations is tightest and may therefore be the most fundamental to the internal ISM. This may be of particular use to the community when only some of these quantities can be measured (e.g., galaxies lacking high-resolution dust continuum imaging). We calculate the significance of each correlation as the deviation of the model from no correlation, or a horizontal line in the given parameter space. The results are shown in Figure 7.

We find that the $\lambda_{\text {peak }}$ versus $D_{\mathrm{MS}}$ and $\lambda_{\text {peak }}$ versus sSFR relationships correlate with $3 \sigma$ and $7 \sigma$ significance, respectively. In contrast, the $\lambda_{\text {peak }}$ versus SFR and $\lambda_{\text {peak }}$ versus $\Sigma_{\mathrm{IR}}$ relationships carry an $18 \sigma$ and $24 \sigma$ correlation significance, respectively. Below, we interpret the underlying physics of the correlations.

To investigate whether the extended redshift range of our sources affects our results, we recalculated the correlations based on a reduced sample with $1.44 \leqslant z \leqslant 2.86$, removing the high- $z$ and low $z$ extrema from the sample. At $z<3$, the reduced sample's continuum observations are on the optically thin portion of the RJ tail of dust emission (i.e., rest-frame wavelengths longer than $\sim 200 \mu \mathrm{m}$ ), probing cold dust in the ISM. With the redshift-restricted sample, the $\lambda_{\text {peak }}-\Sigma_{\mathrm{IR}}$ and $\lambda_{\text {peak }}-\mathrm{SFR}$ correlation significances are reduced somewhat to $16 \sigma$ and $18 \sigma$, respectively, while the correlations between $\lambda_{\text {peak }}$ and $D_{\mathrm{MS}}$ and between $\lambda_{\text {peak }}$ and sSFR increase to $4 \sigma$ and $8 \sigma$, respectively. However, the end results are the same: the $\lambda_{\text {peak }}$ correlations with $\Sigma_{\mathrm{IR}}$ and SFR are significantly stronger than those with sSFR and $D_{\mathrm{MS}}$.

$$
\text { 5.2.1. } \lambda_{\text {peak }} \text { versus } \Sigma_{I R}
$$

We empirically measure the following relationship between $\lambda_{\text {peak }}$ and $\Sigma_{\mathrm{IR}}$, or the star formation surface density, using our sample:

$$
\begin{aligned}
\log \left(\Sigma_{\mathrm{IR}} /\left[L_{\odot} \mathrm{kpc}^{-2}\right]\right)= & (18.78 \pm 0.33) \\
& -(3.80 \pm 0.16) \log \left(\lambda_{\text {peak }} / \mu \mathrm{m}\right) .
\end{aligned}
$$

The overall strength of the correlation is $24 \sigma$. The slope of this relation is a $2.4 \sigma$ deviation from expectation in Equation (5), and the intercept deviates by $4.3 \sigma$. Comparing to the expectation using 

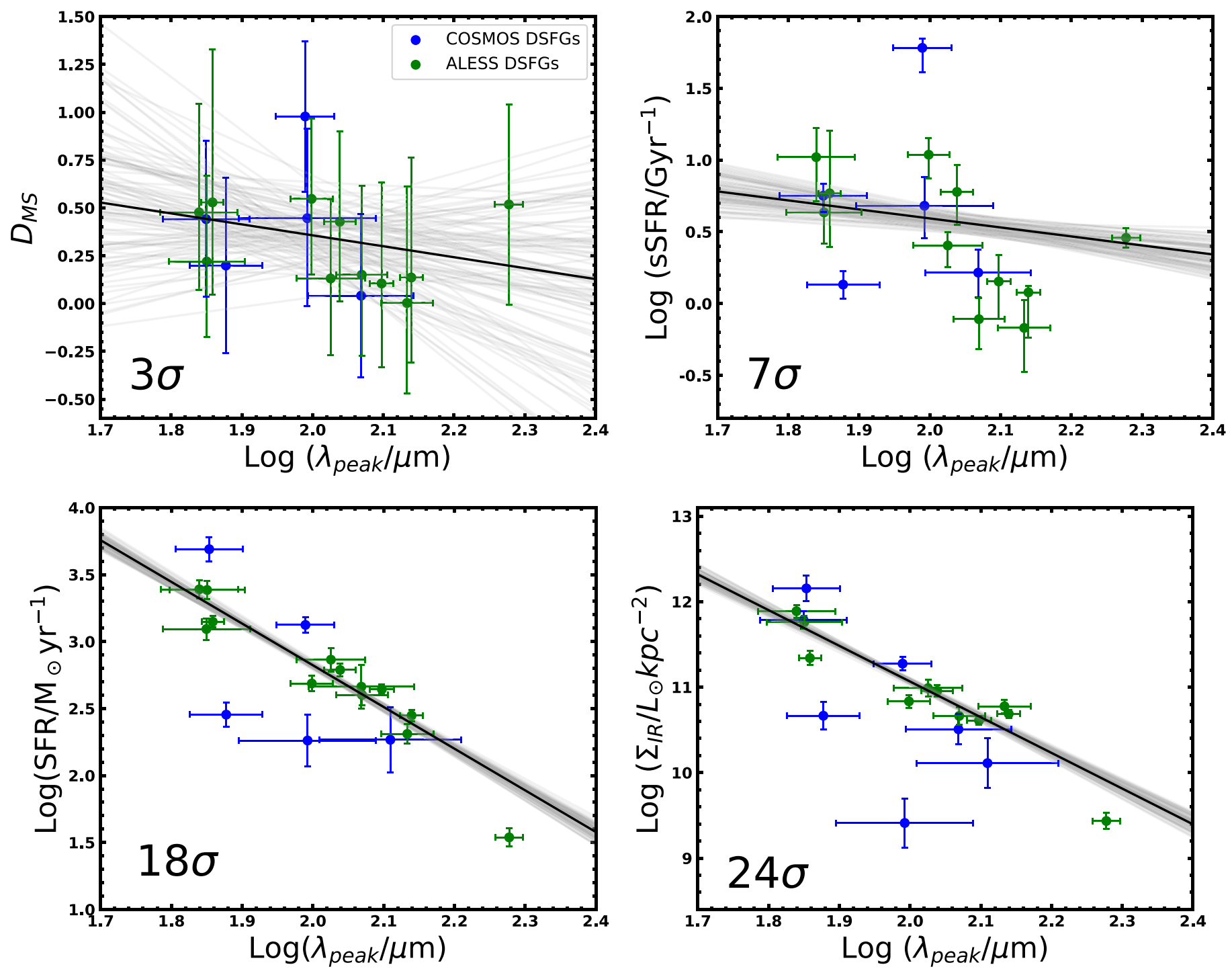

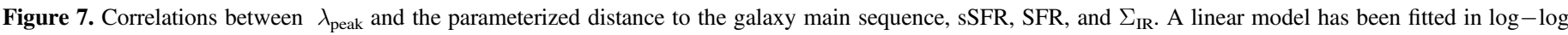
space using MCMC techniques. A representative sample of MCMC trial fits are shown in gray, while the black line shows the best fit. The number in the lower left corner of each plot represents the significance of the relation from a horizontal line, which would indicate no correlation. Larger numbers represent stronger correlations. We infer weak correlations of $\lambda_{\text {peak }}$ with $D_{\mathrm{MS}}$ and SSFR and strong correlations of $\lambda_{\text {peak }}$ with SFR and star formation surface density.

Wien's law directly (Equation (6)), our empirical relation only deviates by $1.3 \sigma$ in the slope and $0.7 \sigma$ in the intercept.

The agreement between Equations (5)-(6) and Equation (7) should be somewhat surprising, given that the geometry of galaxies' ISM is quite complex, and that the ISM is far from optically thick! How might we interpret the remarkably close correlation between $\lambda_{\text {peak }}$ and $\Sigma_{\mathrm{IR}}$ ?

Such a tight correlation would be expected if the peak of dust luminosity (traced directly by $\lambda_{\text {peak }}$ ) originates from regions of the ISM that are, indeed, optically thick. This may not be in direct conflict with more diffuse, cold dust in galaxies' ISM being optically thin, as that dust may not directly dominate the bulk of emission at the peak of IR emission.

We note that Simpson et al. (2017) provide an analysis of several (other) high- $z$ DSFGs that have resolved ALMA sizes and measured SED dust temperatures; their Figure 5 shows the relationship between $T_{\mathrm{d}}$ and $\Sigma_{\mathrm{IR}}$, suggesting tension between predictions from the Stefan-Boltzmann law and galaxies' dust temperatures, when assumed to be optically thick (they find more agreement when using the best-fit temperatures assuming optically thin SEDs). The difference in interpretation between our work and that of Simpson et al. is the presumed normalization of the Stefan-Boltzmann law itself and the geometry of dust: their application of the Stefan-Boltzmann law is drawn from a presumed spherical shell of dust surrounding a single star-forming region (as in Equation (2)), while our normalization accounts for a more disk-like configuration of a dust screen with several spatially distinct deeply embedded star-forming regions (as in Equation (3)). After accounting for the different geometries, we suggest that the Simpson et al. results, like ours, are quite consistent with a galaxy-scale Stefan-Boltzmann approximation for the emergent IR luminosity. Given the strength of the correlation in our data set and the plausible underlying physical interpretation of this relation, we determine that the $\lambda_{\text {peak }}$ versus $\Sigma_{\mathrm{IR}}$ relation is the most fundamental of those we explore in this paper.

We note that the correlation strength of $24 \sigma$ found for $\lambda_{\text {peak }}-\Sigma_{\mathrm{IR}}$ is unaffected by calculating $\Sigma_{\mathrm{IR}}$ with circularized radii or effective radii from Sérsic fitting rather than FWHMs from Gaussian fitting. The strengths of the correlations are also 
not affected by removing from our analysis the two sources (450.03 and 450.14) for which we do not have reliable stellar mass estimates from our analysis.

It is important to note that our measured sizes trace the dust mass as probed on the RJ tail of blackbody emission in what is expected to be an optically thin regime. This may be a different size than would be probed at the peak SED, which traces SFR density more directly. In other words, one might not expect the mass-weighted size to map directly to the SFR-weighted size. The relative agreement of the two is suggestive of a linear relationship between the effective radii over which the dust mass and star formation exist (i.e., $R_{\mathrm{e}}$ for SFR is similar to $R_{\mathrm{e}}$ for $M_{\text {dust }}$ ), which in turn may imply a linear slope for the Kennicutt-Schmidt law of SFR and gas surface density in these galaxies (Kennicutt 1998; de los Reyes \& Kennicutt 2019). Further high-resolution follow-up work is needed to explore this in greater detail.

$$
\text { 5.2.2. } \lambda_{\text {peak }} \text { versus } \mathrm{L}_{I R} \text { or } S F R
$$

We measure the following empirical relationship between $L_{\mathrm{IR}}$ and $\lambda_{\text {peak }}$ in this sample:

$$
\log \left(L_{\mathrm{IR}} / L_{\odot}\right)=(18.2 \pm 0.3)-(2.81 \pm 0.16) \log \left(\lambda_{\text {peak }} / \mu \mathrm{m}\right) .
$$

This correlation carries an overall significance of $18 \sigma$. The relationship with SFR can then be derived using the Kennicutt \& Evans (2012) scaling. Figure 7 demonstrates that the trend broadly follows that of the star formation or IR luminosity surface density, $\Sigma_{\mathrm{IR}}$.

We note that this relation is quite a bit steeper than found elsewhere in the literature for much larger samples of galaxies lacking high-resolution dust observations (e.g., Lee et al. 2013; Symeonidis et al. 2013; Kirkpatrick et al. 2017); for example, Casey et al. (2018b) modeled the $L_{\mathrm{IR}}-\lambda_{\text {peak }}$ relationship by a power law such that $\log _{10}\left(\lambda_{\text {peak }}\right)=\log \left(\lambda_{0}\right)+\eta\left(\log \left(L_{\mathrm{IR}}\right)-12\right)$. Through empirical measurement of large samples of IR-luminous galaxies from $0<z<5$, they found $\eta=-0.068 \pm 0.001$ and $\log \left(\lambda_{0} / \mu \mathrm{m}\right)=2.012$. Though our findings here and the Casey et al. findings both show the same general trend-whereby higherluminosity sources are hotter-here we find that the higherluminosity sources are substantially hotter than those with fainter luminosities. As touched on earlier in Section 3.3, this is likely due to our selection of sources well-suited for high-resolution dust continuum follow-up (and therefore relatively high $L_{\mathrm{IR}}$ for a wide swath of $\lambda_{\text {peak }}$ ); therefore, it is likely that the more extensive samples from Casey et al. (2018a, and references therein) provide a more well-calibrated measurement of the $\lambda_{\text {peak }}-L_{\mathrm{IR}}$ relationship over a much larger dynamic range in $L_{\mathrm{IR}}$.

Because the Casey et al. (2018a) model does not account for galaxy size as our analysis in Equation (7) does, we can use the two in conjunction to infer an overall size dependency of $L_{\mathrm{IR}}$. Combining our Equation (7) with their $\lambda_{\text {peak }}-L_{\mathrm{IR}}$ relationship, we find that the size dependence can be modeled as a powerlaw function of $L_{\mathrm{IR}}$ such that $R_{\mathrm{e}} \propto L_{\mathrm{IR}}^{0.37 \pm 0.03}$. Note that this is roughly consistent with the size-luminosity relationship derived in Fujimoto et al. (2017), who found $R_{\mathrm{e}} \propto L_{\mathrm{IR}}^{0.28 \pm 0.07}$.

\subsection{3. $\lambda_{\text {peak }}$ versus sSFR or Distance to the Main Sequence}

Because both sSFR and the distance of a galaxy from the SFR $-M_{\star}$ relation (the galaxy main sequence) both fundamentally trace physics dependent on both SFR and stellar mass, we discuss them here together. While we do find a subtle relationship between $\lambda_{\text {peak }}$ versus sSFR and $\lambda_{\text {peak }}$ versus $D_{\mathrm{MS}}$, they are substantially weaker than direct correlations between $\lambda_{\text {peak }}$ and $\Sigma_{\mathrm{IR}}$ or $L_{\mathrm{IR}}$.

In contrast to some literature claims, we do not find clear evidence to support the theory that galaxies' dust temperatures correlate strongly with a galaxy's position on the galaxy main sequence. We note that the dynamic range of sSFR and $D_{\mathrm{MS}}$ in our sample is comparable to similar data sets at matched redshifts $\left(\sim 2\right.$ dex in SSFR and $\sim 1$ dex in $D_{\mathrm{MS}}$; Barger et al. 2014). Specifically, we find the following correlations between $\lambda_{\text {peak }}$ and sSFR or $D_{\mathrm{MS}}$ (where we have defined the latter as the orthogonal shortest distance to the Speagle et al. 2014 fits at the source's redshift):

$$
\begin{aligned}
\log \left(\mathrm{sSFR} /\left[\mathrm{Gyr}^{-1}\right]\right)= & (3.4 \pm 0.4) \\
& -(1.43 \pm 0.20) \log \left(\lambda_{\text {peak }} / \mu \mathrm{m}\right)
\end{aligned}
$$

and

$$
\log \left(D_{\mathrm{MS}}\right)=(1.5 \pm 0.4)-(0.54 \pm 0.21) \log \left(\lambda_{\text {peak }} / \mu \mathrm{m}\right),
$$

which have overall correlation significance of $7 \sigma$ and $3 \sigma$, respectively. Note that alternate definitions of $D_{\mathrm{MS}}$ $\left(\Delta \log (\mathrm{SSFR})_{\mathrm{MS}}\right.$ and/or $D_{\mathrm{MS}}$ using the Whitaker et al. 2014 quadratic form of the main sequence) result in similarly low correlation strengths ranging from $1 \sigma$ to $4 \sigma$.

The fact that both correlations between dust temperature and sSFR or $D_{\text {MS }}$ are relatively weak suggests that the inclusion of stellar mass effectively dilutes the underlying physics driving the observed value of $\lambda_{\text {peak }}$. In fact, if we explicitly test for correlation between stellar mass and $\lambda_{\text {peak }}$, indeed, we find no significant correlation.

With integrated dust SED temperature lacking strong direct correlation with the main sequence, if the assertion is made that the main sequence informs on the evolutionary stage of a galaxy, then we infer that $T_{\mathrm{d}}$ (or $\lambda_{\text {peak }}$ ) is not a viable indicator of evolutionary class (starburst or secular disk) and that higher temperature does not necessarily indicate the presence of a starburst (Bothwell et al. 2010; Hodge et al. 2012; Drew et al. 2020). Rather, our results suggest that the underlying dust geometry within the ISM plays a much more substantial role. In addition, ISM dust can be heated by active galactic nuclei whether or not the galaxy hosts an ongoing starburst (Kirkpatrick et al. 2012). Additional recent cosmological simulation work supports the production of intense luminosities through secularly evolving disk systems from either gas infall, minor mergers, or disk instabilities (Davé et al. 2010; Hodge et al. 2012; Narayanan et al. 2015; Hayward et al. 2018; Tadaki et al. 2018).

Magnelli et al. (2014) arrived at different results than we have, suggesting significant correlation between $D_{\mathrm{MS}}$ and $T_{\mathrm{d}}$. Instead of using our definition of $D_{\mathrm{MS}}$, they evaluated $D_{\mathrm{MS}}$ to be $\Delta \log (\mathrm{SSFR})_{\mathrm{MS}}$, where $\Delta \log (\mathrm{SSFR})_{\mathrm{MS}}=\log \left[\mathrm{SSFR} / \mathrm{SSFR}_{\mathrm{MS}}\left(M_{\star}, z\right)\right]$. We also investigated the correlation between $\Delta \log (\mathrm{SSFR})_{\mathrm{MS}}$ and $\lambda_{\text {peak }}$ for our sample and found it to have the same correlation strength as the correlation between $D_{\mathrm{MS}}$, as defined above, and $\lambda_{\text {peak. }}$ Without uncertainties on individual measurements for $\Delta \log (\text { SSFR })_{\mathrm{MS}}$ in Magnelli et al. (2014), we are unable to directly compare those results to our analysis on the relative significance of the correlation. We also note that the stacking techniques used in their analysis on Herschel data may be prone to temperaturedependent biases. For instance, the stacking results of Viero et al. (2013) result in fundamentally different (and overall colder) SEDs 
than the stacking results of Béthermin et al. (2015) despite the use of very similar data sets (P. M. Drew et al. 2021, in preparation). Further analysis of SEDs drawn from broad literature sources is beyond the scope of this work.

\subsection{Comparison to Local Galaxies}

Note that our findings - that $\Sigma_{\mathrm{IR}}$ correlates most closely with $\lambda_{\text {peak }}$ of a dust SED-is in agreement with previous work at lower redshifts, for which a relationship between the star formation surface density, $\Sigma_{\mathrm{IR}}$, and dust temperature exists (Lehnert \& Heckman 1996; Chanial et al. 2007; Lutz et al. 2016; see also Díaz-Santos et al. 2010, which shows that nearby galaxies with higher $L_{\mathrm{IR}}$ have distinctly more compact sizes). While the results of Chanial et al. (2007) find a relatively shallow relationship between $\Sigma_{\mathrm{IR}}$ and $T_{\mathrm{d}}$, our results are more consistent with the earlier (steeper) dependence suggested by Lehnert \& Heckman (1996); the relative discrepancies could be due to measurement of sizes conducted in radio continuum versus millimeter continuum (of which the literature suggests a discrepancy; e.g., Miettinen et al. 2017) or differences in the sample selection.

In particular, we find it useful to analyze the results of Lutz et al. (2016) in context, which is based on FIR size measurements from Herschel/PACS of local galaxies; they derived relationships between $\Sigma_{\mathrm{IR}}, L_{\mathrm{IR}}$, and $\mathrm{sSFR} / \mathrm{sSFR}_{\mathrm{MS}}$ and a proxy measure of dust temperature, the FIR color $\log \left(S_{70} / S_{160}\right)$, which we will call $C$. Their Table 2 provides a summary of the derived relationships, allowing us to directly infer the relative correlations between the FIR color $C$ and $\Sigma_{\mathrm{IR}}$, $L_{\mathrm{IR}}$, and $\Delta \log (\mathrm{SSFR})_{\mathrm{MS}}$ (even though FIR color $C$ is not equivalent to $\lambda_{\text {peak }}$ ). What the Lutz et al. scaling relations suggest is that $C$ and $\Sigma_{\mathrm{IR}}$ are correlated with a measurement significance of $30 \sigma, C$ and $L_{\mathrm{IR}}$ are correlated with a measurement significance of $20 \sigma$, and $C$ and $\Delta \log (\text { SSFR })_{M S}$ are correlated with a measurement significance of $6 \sigma$. While the exact significance of the correlations is likely dependent on sample details, the overall trend is similar to our findings: that $\Sigma_{\text {IR }}$ is the most closely linked physical quantity to dust temperature (or $\lambda_{\text {peak }}$ or $C$ ) and that correlation to the galaxies' main sequence (here probed by $\Delta \log (\mathrm{SSFR})_{\mathrm{MS}}$ ) is the weakest of the correlations.

\section{Conclusions}

We used 0 ". 24 resolved $870 \mu \mathrm{m}$ ALMA dust continuum observations to determine the total integrated flux density and size. We used SED fitting to determine $\lambda_{\text {peak }}$ and $L_{\mathrm{IR}}$. We investigated correlations of dust temperature with four galaxy characteristics: sSFR, parameterized distance to the galaxy main sequence $\left(D_{\mathrm{MS}}\right)$, SFR, and star formation surface density measured via $\Sigma_{\mathrm{IR}}$. Our results are as follows:

1. The $R_{\mathrm{e}}$ of our seven new DSFGs range from 2.1 to $12.03 \mathrm{kpc}$ with sizes of the full sample analyzed at $870 \mu \mathrm{m}$ with $\left\langle R_{\mathrm{e}}\right\rangle=3.9 \pm 1.6 \mathrm{kpc}$. The median Sérsic index $n=0.8 \pm 0.4$ implies a non-Gaussian, exponential disk morphology.

2. The correlations of $\lambda_{\text {peak }}$ with sSFR and $D_{\mathrm{MS}}$ are relatively weak. The linear models relating $\log \left(\lambda_{\text {peak }}\right)$ to $\log (\mathrm{sSFR})$ and $\log \left(D_{\mathrm{MS}}\right)$ had strengths of only $7 \sigma$ and $3 \sigma$, respectively.
3. The linear models relating $\log \left(\lambda_{\text {peak }}\right)$ to $\log (\mathrm{SFR})$ and $\log \left(\Sigma_{\mathrm{IR}}\right)$ had strengths of $18 \sigma$ and $24 \sigma$, respectively, confirming that the $\lambda_{\text {peak }}-L_{\mathrm{IR}}$ and $\lambda_{\text {peak }}-\Sigma_{\mathrm{IR}}$ relationships are statistically stronger than the $\lambda_{\text {peak }}-$ SSFR and $\lambda_{\text {peak }}-D_{\text {MS }}$ relationships and more likely probe the underlying physical driver of galaxies' luminosity-weighted dust temperatures.

4. Our results are consistent with measured scaling relations between FIR color, $\Sigma_{\mathrm{IR}}, L_{\mathrm{IR}}$, and $\Delta \log (\mathrm{sSFR})$ for galaxies in the local universe analyzed by Lutz et al. (2016). In particular, both our work and Lutz et al. find that the $\lambda_{\text {peak }}-\Sigma_{\text {IR }}$ relationships are the strongest. This motivates our conclusion that SFR surface density is the fundamental characteristic driving a galaxy's luminosityweighted dust temperature.

We conclude that galaxies' dust emission properties can be well described by a Stefan-Boltzmann-like law, where the luminosity-weighted dust temperature (as measured via $\lambda_{\text {peak }}$ ) goes roughly as $\Sigma_{\mathrm{IR}} \propto \lambda_{\text {peak }}^{-4}$. While there are many physical reasons such a relationship would be expected not to hold (e.g., our measurement of size on the RJ tail of optically thin dust emission, or the fact that overall dust emission is not optically thick throughout the ISM), our finding that it does hold suggests that (a) the bulk of galaxies' IR luminosity can be approximated as optically thick near the SED peak, roughly consistent with a disk or planar geometry, and (b) there is a linear relationship between galaxies' ISM dust-mass-weighted sizes and galaxies' SFR-weighted sizes. Furthermore, we infer that dust temperature is not necessarily a reliable indication of where a galaxy sits on the main sequence and should not be used to infer evolutionary class, as the quantity is most likely to trace the underlying geometry of galaxies' ISM.

We thank the anonymous reviewer for their input. A.D.B. thanks the John W. Cox Endowment for the Advanced Studies in Astronomy for support. C.M.C. thanks the National Science Foundation for support through grants AST-1714528, AST1814034, and AST-2009577, and additionally C.M.C., J.A.Z., and J.S.S. thank the University of Texas at Austin College of Natural Sciences for support. In addition, C.M.C. acknowledges support from the Research Corporation for Science Advancement from a 2019 Cottrell Scholar Award sponsored by IF/THEN, an initiative of Lyda Hill Philanthropies. S.M.M. thanks the National Science Foundation for support through the Graduate Research Fellowship under grant No. DGE-1610403. C.-C.C. acknowledges support from the Ministry of Science and Technology of Taiwan (MOST 109-2112-M-001-016-MY3). This paper makes use of the following ALMA data: ADS/JAO.ALMA \#2015.1.00568.S. ALMA is a partnership of ESO (representing its member states), NSF (USA), and NINS (Japan), together with NRC (Canada), MOST and ASIAA (Taiwan), and KASI (Republic of Korea), in cooperation with the Republic of Chile. The Joint ALMA Observatory is operated by ESO, AUI/NRAO, and NAOJ. The National Radio Astronomy Observatory is a facility of the National Science Foundation operated under cooperative agreement by Associated Universities, Inc.

\section{ORCID iDs}

Anne D. Burnham (ib https://orcid.org/0000-0003-0274-2537 Caitlin M. Casey (ib https://orcid.org/0000-0002-0930-6466 Jorge A. Zavala (ib https://orcid.org/0000-0002-7051-1100 
Sinclaire M. Manning (1) https://orcid.org/0000-00030415-0121

Justin S. Spilker (1) https://orcid.org/0000-0003-3256-5615

Scott C. Chapman (1) https://orcid.org/0000-0002-8487-3153

Chian-Chou Chen (1) https://orcid.org/0000-0002-3805-0789

Asantha Cooray (1) https://orcid.org/0000-0002-3892-0190

David B. Sanders (1) https://orcid.org/0000-0002-1233-9998

Nick Z. Scoville $\odot$ https://orcid.org/0000-0002-0438-3323

\section{References}

Barger, A. J., Cowie, L. L., Chen, C. C., et al. 2014, ApJ, 784, 9 Béthermin, M., Daddi, E., Magdis, G., et al. 2015, A\&A, 573, A113 Béthermin, M., Wu, H.-Y., Lagache, G., et al. 2017, A\&A, 607, A89 Biggs, A. D., \& Ivison, R. J. 2008, MNRAS, 385, 893

Blain, A. W., Chapman, S. C., Smail, I., \& Ivison, R. 2004, ApJ, 611, 725

Bothwell, M. S., Chapman, S. C., Tacconi, L., et al. 2010, MNRAS, 405, 219 Bruzual, G., \& Charlot, S. 2003, MNRAS, 344, 1000

Casey, C. M. 2012, MNRAS, 425, 3094

Casey, C. M. 2020, ApJ, 900, 68

Casey, C. M., Berta, S., Béthermin, M., et al. 2012, ApJ, 761, 140

Casey, C. M., Chapman, S. C., Beswick, R. J., et al. 2009, MNRAS, 399, 121

Casey, C. M., Chen, C.-C., Cowie, L. L., et al. 2013, MNRAS, 436, 1919

Casey, C. M., Cooray, A., Killi, M., et al. 2017, ApJ, 840, 101

Casey, C. M., Hodge, J., Zavala, J. A., et al. 2018a, ApJ, 862, 78

Casey, C. M., Narayanan, D., \& Cooray, A. 2014, PhR, 541, 45

Casey, C. M., Zavala, J. A., Spilker, J., et al. 2018b, ApJ, 862, 77

Chanial, P., Flores, H., Guiderdoni, B., et al. 2007, A\&A, 462, 81

Chapman, S. C., Blain, A. W., Ivison, R. J., \& Smail, I. R. 2003a, Natur, 422, 695

Chapman, S. C., Helou, G., Lewis, G. F., \& Dale, D. A. 2003b, ApJ, 588, 186 Chapman, S. C., Smail, I., Blain, A. W., \& Ivison, R. J. 2004a, ApJ, 614, 671 Chapman, S. C., Smail, I., Windhorst, R., Muxlow, T., \& Ivison, R. J. 2004b, ApJ, 611, 732

Chen, C.-C., Smail, I., Swinbank, A. M., et al. 2015, ApJ, 799, 194

Conley, A., Cooray, A., Vieira, J. D., et al. 2011, ApJL, 732, L35

da Cunha, E., Charlot, S., \& Elbaz, D. 2008, MNRAS, 388, 1595

da Cunha, E., Walter, F., Smail, I. R., et al. 2015, ApJ, 806, 110

Davé, R., Finlator, K., Oppenheimer, B. D., et al. 2010, MNRAS, 404, 1355

de los Reyes, M. A. C., \& Kennicutt, R. C., Jr. 2019, ApJ, 872, 16

Díaz-Santos, T., Charmandaris, V., Armus, L., et al. 2010, ApJ, 723, 993

Drew, P. M., Casey, C. M., Burnham, A. D., et al. 2018, ApJ, 869, 58

Drew, P. M., Casey, C. M., Cooray, A., \& Whitaker, K. E. 2020, ApJ, 892, 104

Dudzevičiūtè, U., Smail, I., Swinbank, A. M., et al. 2020, MNRAS, 494, 3828

Elbaz, D., Leiton, R., Nagar, N., et al. 2018, A\&A, 616, A110

Fujimoto, S., Ouchi, M., Shibuya, T., \& Nagai, H. 2017, ApJ, 850, 83
Gruppioni, C., Pozzi, F., Rodighiero, G., et al. 2013, MNRAS, 432, 23

Gullberg, B., Smail, I., Swinbank, A. M., et al. 2019, MNRAS, 490, 4956

Hainline, L. J., Blain, A. W., Smail, I., et al. 2011, ApJ, 740, 96

Hayward, C. C., Chapman, S. C., Steidel, C. C., et al. 2018, MNRAS, 476,2278

Hodge, J. A., Carilli, C. L., Walter, F., et al. 2012, ApJ, 760, 11

Hodge, J. A., Riechers, D., Decarli, R., et al. 2015, ApJL, 798, L18

Hodge, J. A., Swinbank, A. M., Simpson, J. M., et al. 2016, ApJ, 833, 103

Jiménez-Andrade, E. F., Zavala, J. A., Magnelli, B., et al. 2020, ApJ, 890, 171

Kennicutt, R. C., \& Evans, N. J. 2012, ARA\&A, 50, 531

Kennicutt, R. C., Jr. 1998, ApJ, 498, 541

Kirkpatrick, A., Pope, A., Alexander, D. M., et al. 2012, ApJ, 759, 139

Kirkpatrick, A., Pope, A., Sajina, A., et al. 2017, ApJ, 843, 71

Kroupa, P., \& Weidner, C. 2003, ApJ, 598, 1076

Laigle, C., McCracken, H. J., Ilbert, O., et al. 2016, ApJS, 224, 24

Lang, P., Schinnerer, E., Smail, I., et al. 2019, ApJ, 879, 54

Lee, N., Sanders, D. B., Casey, C. M., et al. 2013, ApJ, 778, 131

Lehnert, M. D., \& Heckman, T. M. 1996, ApJ, 472, 546

Li, A., \& Draine, B. T. 2001, ApJ, 554, 778

Lutz, D., Berta, S., Contursi, A., et al. 2016, A\&A, 591, A136

Magdis, G. E., Daddi, E., Béthermin, M., et al. 2012, ApJ, 760, 6

Magnelli, B., Lutz, D., Saintonge, A., et al. 2014, A\&A, 561, A86

Michałowski, M. J., Dunlop, J. S., Cirasuolo, M., et al. 2012, A\&A, 541, A85

Miettinen, O., Delvecchio, I., Smolčić, V., et al. 2017, A\&A, 606, A17

Narayanan, D., Turk, M., Feldmann, R., et al. 2015, Natur, 525, 496

Peng, C. Y., Ho, L. C., Impey, C. D., \& Rix, H.-W. 2010, AJ, 139, 2097

Perault, M. 1987, PhD thesis, Univ. Paris

Roseboom, I. G., Dunlop, J. S., Cirasuolo, M., et al. 2013, MNRAS, 436, 430

Rujopakarn, W., Dunlop, J. S., Rieke, G. H., et al. 2016, ApJ, 833, 12

Sajina, A., Yan, L., Armus, L., et al. 2007, ApJ, 664, 713

Sanders, D. B., Mazzarella, J. M., Kim, D.-C., Surace, J. A., \& Soifer, B. T. 2003, AJ, 126, 1607

Schreiber, C., Elbaz, D., Pannella, M., et al. 2018, A\&A, 609, A30

Siebenmorgen, R., \& Krügel, E. 2007, A\&A, 461, 445

Simpson, J. M., Smail, I., Swinbank, A. M., et al. 2017, ApJ, 839, 58

Speagle, J. S., Steinhardt, C. L., Capak, P. L., \& Silverman, J. D. 2014, ApJS, 214,15

Spilker, J. S., Marrone, D. P., Aravena, M., et al. 2016, ApJ, 826, 112

Swinbank, A. M., Simpson, J. M., Smail, I., et al. 2014, MNRAS, 438, 1267

Symeonidis, M., Vaccari, M., Berta, S., et al. 2013, MNRAS, 431, 2317

Tadaki, K., Iono, D., Yun, M. S., et al. 2018, Natur, 560, 613

U, V., Sanders, D. B., Mazzarella, J. M., et al. 2012, ApJS, 203, 9

Viero, M. P., Moncelsi, L., Quadri, R. F., et al. 2013, ApJ, 779, 32

Whitaker, K. E., Franx, M., Leja, J., et al. 2014, ApJ, 795, 104

Wien, W. 1897, The London, Edinburgh, and Dublin Philosophical Magazine and Journal of Science, 43, 214

Younger, J. D., Omont, A., Fiolet, N., et al. 2009, MNRAS, 394, 1685 